\title{
Human in-vivo brain magnetic resonance current density imaging (MRCDI)
}

\author{
Göksu, Cihan; Hanson, Lars G. ; Siebner, Hartwig R; Ehses, Philipp; Scheffler, Klaus; Thielscher, Axel
}

Published in:

Neurolmage

Link to article, DOI:

10.1016/j.neuroimage.2017.12.075

Publication date:

2017

Document Version

Peer reviewed version

Link back to DTU Orbit

Citation (APA):

Göksu, C., Hanson, L. G., Siebner, H. R., Ehses, P., Scheffler, K., \& Thielscher, A. (2017). Human in-vivo brain magnetic resonance current density imaging (MRCDI). Neurolmage, 171, 26-39.

https://doi.org/10.1016/j.neuroimage.2017.12.075

\section{General rights}

Copyright and moral rights for the publications made accessible in the public portal are retained by the authors and/or other copyright owners and it is a condition of accessing publications that users recognise and abide by the legal requirements associated with these rights.

- Users may download and print one copy of any publication from the public portal for the purpose of private study or research.

- You may not further distribute the material or use it for any profit-making activity or commercial gain

- You may freely distribute the URL identifying the publication in the public portal

If you believe that this document breaches copyright please contact us providing details, and we will remove access to the work immediately and investigate your claim 


\section{Accepted Manuscript}

Human in-vivo brain magnetic resonance current density imaging (MRCDI)

Cihan Göksu, Lars G. Hanson, Hartwig R. Siebner, Philipp Ehses, Klaus Scheffler, Axel Thielscher

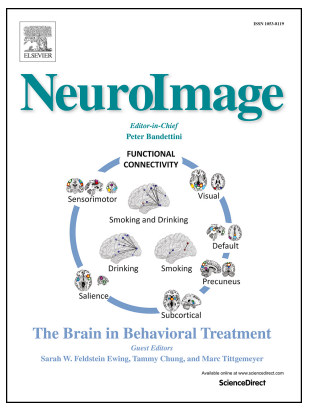

PII: S1053-8119(17)31099-6

DOI: $\quad$ 10.1016/j.neuroimage.2017.12.075

Reference: YNIMG 14590

To appear in: Neurolmage

Received Date: 1 November 2017

Accepted Date: 22 December 2017

Please cite this article as: Göksu, C., Hanson, L.G., Siebner, H.R., Ehses, P., Scheffler, K., Thielscher, A., Human in-vivo brain magnetic resonance current density imaging (MRCDI), Neurolmage (2018), doi: 10.1016/j.neuroimage.2017.12.075.

This is a PDF file of an unedited manuscript that has been accepted for publication. As a service to our customers we are providing this early version of the manuscript. The manuscript will undergo copyediting, typesetting, and review of the resulting proof before it is published in its final form. Please note that during the production process errors may be discovered which could affect the content, and all legal disclaimers that apply to the journal pertain. 
${ }^{1}$ Danish Research Centre for Magnetic Resonance, Centre for Functional and Diagnostic Imaging and Research, Copenhagen University Hospital Hvidovre, Denmark.

2 Center for Magnetic Resonance, DTU Elektro, Technical University of Denmark, Kgs Lyngby, Denmark.

${ }^{3}$ High-Field Magnetic Resonance Center, Max-Planck-Institute for Biological Cybernetics, Tübingen, Germany.

${ }^{4}$ Department of Biomedical Magnetic Resonance, University of Tübingen, Tübingen, Germany.

${ }^{5}$ Department of Neurology, Copenhagen University Hospital, Bispebjerg, Denmark.

${ }^{6}$ German Center for Neurodegenerative Diseases (DZNE), Bonn, Germany

$\dagger$ These authors contributed equally to this work.

6

7

8

9

10

11

12

Klaus Scheffler ${ }^{3,4 \dagger}$, and Axel Thielscher ${ }^{1,2,3 \dagger^{*}}$

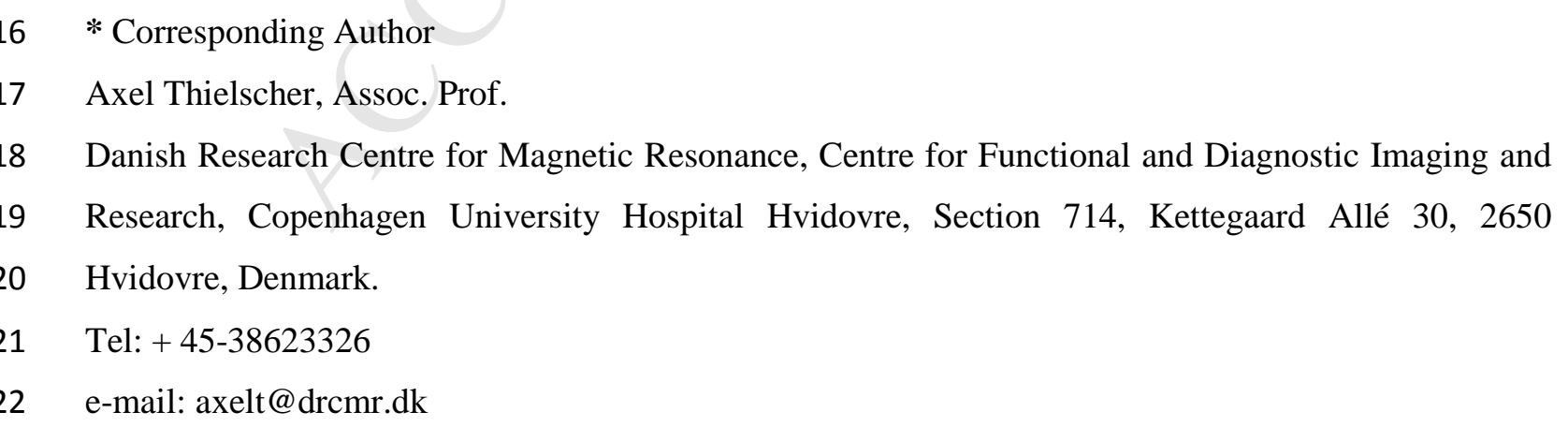

\section{Human In-vivo Brain Magnetic Resonance Current Density Imaging (MRCDI)}


23

24

25

26

27

28

29

30

\section{ABSTRACT}

Magnetic resonance current density imaging (MRCDI) and MR electrical impedance tomography (MREIT) are two emerging modalities, which combine weak time-varying currents injected via surface electrodes with magnetic resonance imaging (MRI) to acquire information about the current flow and ohmic conductivity distribution at high spatial resolution. The injected current flow creates a magnetic field in the head, and the component of the induced magnetic field $\Delta \mathrm{B}_{z, \mathrm{c}}$ parallel to the main scanner field causes small shifts in the precession frequency of the magnetization. The measured MRI signal is modulated by these shifts, allowing to determine $\Delta \mathrm{B}_{\mathrm{z}, \mathrm{c}}$ for the reconstruction of the current flow and ohmic conductivity.

Here, we demonstrate reliable $\Delta \mathrm{B}_{z, \mathrm{c}}$ measurements in-vivo in the human brain based on multi-echo spin echo (MESE) and steady-state free precession free induction decay (SSFP-FID) sequences. In a series of experiments, we optimize their robustness for in-vivo measurements while maintaining a good sensitivity to the current-induced fields. We validate both methods by assessing the linearity of the measured $\Delta \mathrm{B}_{z, \mathrm{c}}$ with respect to the current strength. For the more efficient SSFP-FID measurements, we demonstrate a strong influence of magnetic stray fields on the $\Delta \mathrm{B}_{\mathrm{z}, \mathrm{c}}$ images, caused by non-ideal paths of the electrode cables, and validate a correction method. Finally, we perform measurements with two different current injection profiles in five subjects. We demonstrate reliable recordings of $\Delta \mathrm{B}_{\mathrm{z}, \mathrm{c}}$ fields as weak as $1 \mathrm{nT}$, caused by currents of $1 \mathrm{~mA}$ strength. Comparison of the $\Delta \mathrm{B}_{\mathrm{z}, \mathrm{c}}$ measurements with simulated $\Delta \mathrm{B}_{\mathrm{z}, \mathrm{c}}$ images based on FEM calculations and individualized head models reveals significant linear correlations in all subjects, but only for the stray field-corrected data. As final step, we reconstruct current density distributions from the measured and simulated $\Delta \mathrm{B}_{\mathrm{z}, \mathrm{c}}$ data. Reconstructions from non-corrected $\Delta \mathrm{B}_{\mathrm{z}, \mathrm{c}}$ measurements systematically overestimate the current densities. Comparing the current densities reconstructed from corrected $\Delta \mathrm{B}_{\mathrm{z}, \mathrm{c}}$ measurements and from simulated $\Delta \mathrm{B}_{z, \mathrm{c}}$ images reveals an average coefficient of determination $\mathrm{R}^{2}$ of $71 \%$. In addition, it shows that the simulations underestimated the current strength on average by $24 \%$.

Our results open up the possibility of using MRI to systematically validate and optimize numerical field simulations that play an important role in several neuroscience applications, such as transcranial brain stimulation, and electro- and magnetoencephalography.

\section{Key words:}

Current-induced magnetic field, magnetic resonance current density imaging, multi-echo spin echo, steady-state free precession free induction decay, in-vivo imaging 


\section{INTRODUCTION}

Accurate knowledge of the current flow distribution in the human head caused by neural or external sources is important in several neuroscience applications such as targeting control in transcranial brain stimulation (TBS) and source localization in electro- (EEG) and magnetoencephalography (MEG) (Mosher et al., 1999; Nitsche and Paulus, 2000). The current distributions are usually derived using forward modeling schemes that employ volume conductor models of the head (Oostenveld et al., 2011; Tadel et al., 2011; Thielscher et al., 2015). However, even anatomically accurate models of the head still suffer from uncertainties of the tissue conductivities. The conductivity values reported in literature vary substantially across studies, likely caused by both methodological differences and natural physiological variability, with the amount of uncertainty depending on the tissue type (Dabek et al., 2016; Faes et al., 1999; Huang et al., 2017; Miranda, 2013). Methods to measure the current flow non-invasively in-vivo are thus important for the validation and improvement of these forward modelling approaches.

Magnetic resonance current density imaging (MRCDI) and MR electrical impedance tomography (MREIT) are two emerging modalities, which combine weak time-varying currents injected via surface electrodes with magnetic resonance imaging (MRI) to acquire information about the current flow and ohmic conductivity at high spatial resolution (Eyüboğlu, 2006a, 2006b; Göksu et al., 2014; Joy, 2004; Scott et al., 1991; Seo and Woo, 2011; Woo et al., 1994). In short, the injected current flow creates a magnetic field in the head, and the component of the induced magnetic field $\Delta \mathrm{B}_{z, c}$ parallel to the main magnetic field of the scanner slightly changes the precession frequency of the magnetization (here, the $\mathrm{z}$-axis is chosen along the static scanner field, and $\Delta \mathrm{B}_{\mathrm{z}, \mathrm{c}}$ is correspondingly the currentinduced field change). This modulates the phase of the measured MRI signal proportional to $\Delta \mathrm{B}_{z, \mathrm{c}}$. The current-induced phase changes can thus be used to determine $\Delta \mathrm{B}_{\mathrm{z}, \mathrm{c}}$, and to reconstruct the inner current flow and the ohmic conductivity distribution (Eyüboğlu, 2006b, 2006c; Ider and Birgül, 1998; Joy, 2004; Oh et al., 2003; Scott et al., 1991; Seo and Woo, 2011).

Up to now, successful MRCDI and MREIT recordings have been demonstrated in phantoms, animal models and in-vivo in human limbs (Birgül et al., 2003; Eyüboğlu, 2006c; Han et al., 2010; Ider and Birgül, 1998; Jeon et al., 2009; Jeong et al., 2010; Kim et al., 2009, 2008, 2011; Meng et al., 2012; Oh et al., 2005, 2003; Sadighi et al., 2014; Sadleir et al., 2005; Seo and Woo, 2011; Woo and Seo, 2008). However, in order to achieve a sufficient signal-to-noise ratio (SNR) of the $\Delta \mathrm{B}_{\mathrm{z}, \mathrm{c}}$ images, these studies applied current strengths that were much higher than those applicable for in-vivo human brain applications (1-2 mA; Utz et al., 2010). Only recently, the first proof-of-principle studies have been performed that demonstrated the feasibility of acquiring $\Delta \mathrm{B}_{z, \mathrm{c}}$ images for the human brain in-vivo using weak current strengths (Jog et al., 2016; Kasinadhuni et al., 2017). These initial results are promising, but highlight the need for further improvements of the measurement procedures and 
sequences to allow for sufficient quality and unambiguous $\Delta \mathrm{B}_{z, \mathrm{c}}$ images in a reasonable acquisition time.

Using comprehensive theoretical analyses and phantom measurements, we have previously optimized the sensitivity of two MRI sequences for in-vivo MRCDI and MREIT measurements in the human brain (Göksu et al., 2017). We explored multi-echo spin echo (MESE) and steady-state free precession free induction decay (SSFP-FID) sequences, and derived optimized parameters to maximize their efficiency for measuring current-induced phase changes, given relaxation parameters of brain tissue at $3 \mathrm{~T}$. Here, we validate the performance of the optimized sequences for in-vivo brain imaging and improve their robustness to artifacts that are of concern in an in-vivo setting, in order to ensure the validity of the results. Using the adapted approach, we perform measurements with two different current injection profiles in five subjects using SSFP-FID, and demonstrate reliable recordings of $\Delta \mathrm{B}_{z, \mathrm{c}}$ fields as weak as $1 \mathrm{nT}$. We compare the $\Delta \mathrm{B}_{z, \mathrm{c}}$ measurements with simulations based on the Finite-Element Method (FEM) and individualized head models reconstructed from structural MR images of the same subjects. As final step, we reconstruct the current flow distributions from both the measured and simulated $\Delta \mathrm{B}_{\mathrm{z}, \mathrm{c}}$ data. Taken together, the results presented here highlight the importance of careful validation of the measurement procedures to ensure unambiguous current density reconstructions. They optimize the novel $\Delta \mathrm{B}_{\mathrm{z}, \mathrm{c}}$ measurements for in-vivo applications, and pave the way for their application in future MRCDI and MREIT studies of the human brain.

\section{METHODS}

\section{Subjects}

Thirteen healthy subjects were included in the study, which consisted of five successive experiments. Five participants took part in two of the experiments, and two participated three times. They had no previous history of neurological or psychiatric disorders and were screened for contraindications to MRI and TBS. Written informed consent was obtained from all participants prior to the scans. The study complied with the Helsinki declaration on human experimentation and was approved by the Ethics Committee of the Capital Region of Denmark (H16032361).

\section{Sequence of Experiments}

Our study is organized in five successive experiments:

- First, we compare the SNR and quality of $\Delta \mathrm{B}_{z, \mathrm{c}}$ images acquired with single- vs. multi-gradientecho readouts. Our prior results demonstrated the need to use long echo times for MESE and long repetition times for SSFP-FID in order to maximize efficiency. The resulting SNR-optimal low readout bandwidth (BW) decreases image quality as it causes considerable distortions particularly for in-vivo applications. Here, we test to which extent these effects can be prevented by using 
multi-gradient-echo readouts that are acquired at a higher BW and that are subsequently combined to reconstruct the $\Delta \mathrm{B}_{z, \mathrm{c}}$ image.

- Second, we validate the methods by assessing the linearity of the measured $\Delta \mathrm{B}_{\mathrm{z}, \mathrm{c}}$ with respect to the strength of the injected currents.

- Third, we focus on the more efficient SSFP-FID measurements and assess the influence of magnetic stray fields on the $\Delta \mathrm{B}_{z, \mathrm{c}}$ images, caused by non-ideal paths of the feeding cables that are connected to the electrodes. We propose and validate a method to correct for these undesired influences.

- Fourth, we re-evaluate the impact of the chosen repetition times on measurement efficiency and image quality in the presence of physiological noise. We test whether decreasing the repetition times below the theoretically optimal values can help to improve image quality without substantially sacrificing the SNR of the $\Delta \mathrm{B}_{\mathrm{z}, \mathrm{c}}$ images.

- Fifth, we perform $\Delta \mathrm{B}_{\mathrm{z}, \mathrm{c}}$ measurements with two different current injection profiles (right-left and anterior-posterior), and compare the measurements with simulations based on the Finite-Element Method (FEM) and individualized head models reconstructed from structural MR images of the same subjects. We also reconstruct and compare the current density distributions from the measured and simulated $\Delta \mathrm{B}_{z, \mathrm{c}}$ data. For both the $\Delta \mathrm{B}_{z, \mathrm{c}}$ images and current flow distributions, we test how much the correction of the cable-induced magnetic stray fields affects the similarity between measured and simulated data.

\section{MRI sequences for MRCDI}

We tested the in-vivo application of two different MRCDI sequences, MESE (Fig. 1a) and SSFP-FID (Fig. 1b). Details of the sequences can be found in (Göksu et al., 2017). In short, we selected MESE because of its high SNR for the magnitude images and its robustness to field inhomogeneity (Nam and Kwon, 2010), and SSFP-FID for its high phase sensitivity (Lee et al., 2016; Scheffler et al., 2006).

For MESE (Fig. 1a), the measured current-induced magnetic field for the $\mathrm{n}^{\text {th }}$ spin-echo $\Delta \mathrm{B}_{\mathrm{z}, \mathrm{c}}^{\mathrm{n}}$ is given as

$$
\Delta \mathrm{B}_{\mathrm{z}, \mathrm{c}}^{\mathrm{n}}=\left(\angle \mathrm{M}_{\mathrm{n}}^{+}-\angle \mathrm{M}_{\mathrm{n}}^{-}\right) / 2 \gamma \mathrm{T}_{\mathrm{ES}} \mathrm{n},
$$

where $\gamma$ is the gyromagnetic ratio of protons, and $\mathrm{T}_{\mathrm{ES}}$ the echo spacing. The measurement is performed twice with opposite current injection profiles, and $\angle \mathrm{M}_{\mathrm{n}}^{+}$and $\angle \mathrm{M}_{\mathrm{n}}^{-}$are the phases of the acquired complex MR images for the positive and negative current directions for the $\mathrm{n}^{\text {th }}$ echo (Göksu et al., 2017; Nam and Kwon, 2010; Scott et al., 1992). The final $\Delta B_{z, c}$ image is determined as the weighted sum of the $\Delta \mathrm{B}_{\mathrm{z}, \mathrm{c}}^{\mathrm{n}}$ images of the single echoes, with the weightings being proportional to the inverse of the variances of the images (Göksu et al., 2017). When a multi-gradient-echo readout is 
used, the final $\Delta \mathrm{B}_{z, \mathrm{c}}$ image is determined in the same way after summation across all acquired gradient echoes.

For SSFP-FID (Fig. 1b), the $\Delta \mathrm{B}_{\mathrm{z}, \mathrm{c}}$ image in case of weak injection currents is given as

$$
\Delta \mathrm{B}_{\mathrm{z}, \mathrm{c}}=\frac{\angle \mathrm{M}_{\mathrm{SS} 1}-\angle \mathrm{M}_{\mathrm{SS} 2}}{\mathrm{~m}_{\mathrm{seq}}},
$$

with $\angle \mathrm{M}_{\mathrm{SS} 1}$ and $\Delta \mathrm{M}_{\mathrm{SS} 2}$ being the phase images for echoes with positive and negative current injection. The constant $\mathrm{m}_{\text {seq }}=\partial\left(\angle \mathrm{M}_{\mathrm{SS} 1}-\angle \mathrm{M}_{\mathrm{SS} 2}\right) / \partial \Delta \mathrm{B}_{\mathrm{z}, \mathrm{c}}$ is the phase sensitivity to magnetic field changes (Göksu et al., 2017). We calculated it via spin simulations based on 3D rotation and relaxation matrices (Jaynes, 1955), and it depends on the sequence and tissue relaxation parameters.

\section{Measurement procedures}

All experiments were performed on a $3 \mathrm{~T}$ MRI scanner (MAGNETOM Prisma, SIEMENS Healthcare, Erlangen, Germany) equipped with a 64-channel head coil. Multi-channel signals were combined using an adaptive combine algorithm that employs a spatial matched filter created from the individual coil images without a-priori knowledge of coil sensitivity maps (Walsh et al., 2000). The electrical current waveforms were created using a waveform generator (33500B; Keysight Technologies, Santa Clara, CA, USA), amplified using an MR-conditional device for transcranial weak current stimulation (DC-STIMULATOR PLUS, neuroConn GmbH, Ilmenau, Germany), and were applied to the participants via circular rubber electrodes $(5 \mathrm{~cm}$ in diameter $)$ attached to the scalp. We used two different electrode configurations that created current flows either from right to left (RL) or from anterior to posterior (A-P) in the brain. For R-L current injection, the rubber electrodes were attached symmetrically at positions directly above and slightly anterior to the ears using conductive paste (Ten20, Weaver and Company, Colorado, USA). This corresponds roughly to positions above the temporoparietal junctions. For A-P injection, one electrode was placed centrally on the forehead and the second centrally superior to the inion. Unless stated otherwise, peak current amplitudes of $\pm 1 \mathrm{~mA}$ were used. A ramp up period of $10 \mathrm{~s}$ was used in order to prevent sudden subject motion. MR data acquired during this period were discarded.

We used single-slice MESE and SSFP-FID measurements, with an axial slice placed in the upper half of the brain. Based on an initial structural image (details are given below), the slice position was chosen to contain approximately the electrode centers. The fat signal was suppressed by a chemicalshift-selective (CHESS) fat suppression technique (Haase et al., 1985). A field of view (FOV) of $224 \times 180 \mathrm{~mm}^{2}$, an image matrix of $112 \times 90$ and a voxel size of $2 \times 2 \times 3 \mathrm{~mm}^{3}$ were used for both sequence types. For MESE, the echo spacing was $\mathrm{T}_{\mathrm{ES}}=60 \mathrm{~ms}$, repetition time was $\mathrm{T}_{\mathrm{R}}=1.5 \mathrm{~s}$ and the number of spin echoes was $\mathrm{N}_{\mathrm{SE}}=3$. For SSFP-FID, the tip angle was $\alpha=30^{\circ}$. The other MR sequence 
parameters varied across experiments and are stated below. All experiments were performed with both positive and negative current directions (i.e. two subsequent acquisitions of each k-space line; the first corresponds to the positive direction and the second to the negative). The current waveforms were employed as indicated in Figure $1\left(\mathrm{I}_{\mathrm{c}}^{+}\right.$for the first acquisition and $\mathrm{I}_{\mathrm{c}}^{-}$for the second). By that, each k-space line was acquired twice in successive readout periods with opposite currents $\mathrm{I}_{\mathrm{c}}^{+}$and $\mathrm{I}_{\mathrm{c}}^{-}$ to measure two phase images with opposite current-induced phases. After acquisition of the complete $\mathrm{k}$-space, the measurements were repeated. The MESE measurements were repeated twice $\left(\mathrm{N}_{\text {meas }}=2\right)$, with a total scan time of $\mathrm{T}_{\text {tot }} \approx 9$ mins. For SSFP-FID, the number of measurements $\mathrm{N}_{\text {meas }}$ varied across experiments and are stated below. Generally, they were selected as high as possible while limiting the total duration of each experiment for the participants to 2 hours. This included up to 1 hour 20 minutes of MR scanning (in experiment 2; details are stated below), of which maximally 45 minutes were combined with current stimulation.

For all subjects, a high-resolution structural image was acquired using the Pointwise Encoding Time reduction with Radial Acquisition (PETRA) sequence (Ida et al., 2015) with number of slices $\mathrm{N}_{\mathrm{sli}}=$ 320 , image matrix 320x320, voxel size $0.9 \times 0.9 \times 0.9 \mathrm{~mm}^{3}$, tip angle $\alpha=6^{\circ}, \mathrm{T}_{\mathrm{R}}=3.61 \mathrm{~ms}, \mathrm{~T}_{\mathrm{E}}=0.07$ $\mathrm{ms}$, inversion time $\mathrm{T}_{\mathrm{I}}=0.5 \mathrm{~s}, \mathrm{BW}=359 \mathrm{~Hz} /$ pixel, and turbo factor 400 . The images exhibited a $\mathrm{T}_{1^{-}}$ weighted contrast for soft tissue. In addition, it allowed locating the rubber of the electrodes and of the cable insulations due to the short $\mathrm{T}_{\mathrm{E}}$. The visibility of the cable tracks was further improved by covering them with Play-Doh (Hasbro Inc., RI, USA) which provides strong MRI signal due to its high water content.

For participants in which the current flow distribution was estimated using FEM calculations, additional $\mathrm{T}_{1^{-}}$and $\mathrm{T}_{2}$-weighted images were acquired for the construction of individualized volume conductor models. The $\mathrm{T}_{1}$-weighted images were based on a Magnetization-Prepared Rapid Acquisition Gradient-Echo (MPRAGE) sequence with number of slices $\mathrm{N}_{\mathrm{sli}}=208$, image matrix $256 \times 256$, voxel size $1 \times 1 \times 1 \mathrm{~mm}^{3}$, tip angle $\alpha=9^{\circ}, \mathrm{T}_{\mathrm{R}}=2700 \mathrm{~ms}, \mathrm{~T}_{\mathrm{E}}=3.63 \mathrm{~ms}$, and inversion time $\mathrm{T}_{\mathrm{I}}$ $=1090 \mathrm{~ms}$ with selective water excitation. The $\mathrm{T}_{2}$-weighted images used a Sampling Perfection with Application-optimized Contrasts using different flip-angle Evolutions (SPACE) sequence with $\mathrm{N}_{\text {sli }}=$ 208, image matrix 256x256, voxel size $1 \times 1 \times 1 \mathrm{~mm}^{3}, \mathrm{~T}_{\mathrm{R}}=3200 \mathrm{~ms}, \mathrm{~T}_{\mathrm{E}}=408 \mathrm{~ms}$, and turbo factor 282 .

\section{Experiment 1: Single-vs. Multi-gradient-echo acquisition}

In three participants, we compared the quality and SNR of the $\Delta \mathrm{B}_{z, \mathrm{c}}$ images based on multi-gradientecho readouts at high BW versus their single-gradient-echo counterparts at low BW, employing an RL electrode montage. The experiments were performed both with and without current injection. The MESE experiments were repeated for $\mathrm{N}_{\mathrm{GE}}=1(\mathrm{BW}=19.2 \mathrm{~Hz} /$ pixel; echo time point relative to the 
preceding refocusing pulse: $\left.\mathrm{T}_{\mathrm{GE}}=30 \mathrm{~ms}\right)$ and $\mathrm{N}_{\mathrm{GE}}=5(\mathrm{BW}=103.6 \mathrm{~Hz} /$ pixel; gradient echo time points relative to the preceding refocusing pulse: $\left.\mathrm{T}_{\mathrm{GE}}=[8.8,19.6,30,40.6,51.2] \mathrm{ms}\right)$. The SSFP-FID experiments were performed with $\mathrm{T}_{\mathrm{R}}=120 \mathrm{~ms}$ and $\mathrm{N}_{\text {meas }}=12$ ( $\left.\mathrm{T}_{\text {tot }} \approx 4.5 \mathrm{mins}\right)$. They were repeated for $\mathrm{N}_{\mathrm{GE}}=1\left(\mathrm{BW}=12 \mathrm{~Hz} /\right.$ pixel; gradient echo time point relative to the preceding RF pulse: $\mathrm{T}_{\mathrm{GE}}=60$ $\mathrm{ms})$ and $\mathrm{N}_{\mathrm{GE}}=7\left(\mathrm{BW}=75 \mathrm{~Hz} /\right.$ pixel; gradient echo time points: $\mathrm{T}_{\mathrm{GE}}=[8.33,22.43,36.53,50.63$, $64.73,79.00,93.16] \mathrm{ms})$.

MESE was tested at its optimal $\mathrm{T}_{\mathrm{ES}}$ of $60 \mathrm{~ms}$, while SSFP-FID was tested at its optimal $\mathrm{T}_{\mathrm{R}}$ of $120 \mathrm{~ms}$, resulting in a different number of readouts for the multi-gradient-echo cases. For both sequences, the number of readouts was chosen to result in a BW that was high enough to prevent visible distortions. The quality of the resulting $\Delta \mathrm{B}_{z, c}$ images was evaluated by visual inspection. In addition, the performance of the methods was quantified by creating histograms of the noise floor in the $\Delta \mathrm{B}_{z, \mathrm{c}}$ images acquired without current injection. For that, masks created from the magnitude images were used to extract the values from the brain. Gaussian distributions were fitted to the histograms, and the differences in the mean $\mu_{\Delta \mathrm{Bzc}}$ and standard deviation $\sigma_{\Delta \mathrm{Bzc}}$ of the fits were evaluated.

\section{Experiment 2: Linear dependence of the measured $\Delta B_{z, c}$ on current strength}

In order to verify the linear dependence of the measured $\Delta \mathrm{B}_{z, \mathrm{c}}$ on the strength of the injected currents, MESE and SSFP-FID experiments using multi-gradient-echo readouts were performed in four participants. The data of one subject was discarded due to severe motion artefacts. For MESE, $\mathrm{N}_{\mathrm{GE}}=$ 5 was used. The parameters for SSFP-FID were $T_{R}=120 \mathrm{~ms}, \mathrm{~N}_{\text {meas }}=12$ and $\mathrm{N}_{\mathrm{GE}}=7$. For each participant, measurements at four currents strength $\left(I_{c}=0,0.33,0.66\right.$ and $\left.1 \mathrm{~mA}\right)$ were acquired in random order, using an R-L electrode montage. This resulted in $4 \times 12=48$ SSFP-FID and $4 \times 2=8$ MESE measurements per participant. For each measurement, average $\Delta \mathrm{B}_{z, \mathrm{c}}$ values were extracted from a region-of-interest (ROI) that was individually positioned to exhibit clear current-induced phase changes for the MESE measurements at $1 \mathrm{~mA}$. Linear regression models of the extracted $\Delta \mathrm{B}_{\mathrm{z}, \mathrm{c}}$ values as a function of $I_{c}$ were fitted both to the MESE and SSFP-FID results, and the mean shifts $\beta_{0}$ and slopes $\beta_{1}$ and their standard errors are reported.

\section{Experiment 3: Correction of cable-induced stray magnetic fields}

Given the higher efficiency of SSFP-FID compared to MESE (Göksu et al., 2017), we focused on SSFP-FID in the rest of the study. The sequence parameters were $T_{R}=120 \mathrm{~ms}, \mathrm{~N}_{\text {meas }}=24\left(\mathrm{~T}_{\text {tot }} \approx 9\right.$ mins) and $\mathrm{N}_{\mathrm{GE}}=7$. In the proximity of the head, the cables connecting the electrodes to the current stimulator should be fully parallel to the main magnetic field of the scanner. This ensures that the magnetic fields created by the current flow through the cables do not contribute to the phase of the measured MR images. Any deviation from an ideal parallel cable path can result in strong stray fields which change the measured $\Delta \mathrm{B}_{\mathrm{z}, \mathrm{c}}$ distribution. For example, a straight wire of $10 \mathrm{~cm}$ length that 
carries a current of $1 \mathrm{~mA}$ and is placed parallel to an axial imaging plane at a distance of $10 \mathrm{~cm}$ changes the z-component of the magnetic field in the plane by up to $0.9 \mathrm{nT}$. This is approximately the situation encountered if the electrode cables meet just above or below the head, and the resulting field change is similar to that caused by current flow inside the head. However, parallel cable paths are difficult to achieve in practice, as modern multi-channel receive coils fit tightly around the head. Changing to, e.g. birdcage coils would strongly reduce the SNR of the measurements. In addition, in our measurements, the stray fields were severe as we employed a twisted wire pair that branched out only in close proximity to the head. This was caused by the need to employ stimulator equipment that was $\mathrm{CE}$ approved as medical device.

Using SSFP-FID measurements in four participants, we demonstrated the impact of the cable-induced stray fields on the $\Delta \mathrm{B}_{z, \mathrm{c}}$ images. A wire loop was placed around the head, with the upper half following a similar path as the cables in the other measurements. The lower half of the loop was extended inferior, with the wires being as parallel as possible to the main magnetic field for $30 \mathrm{~cm}$ before they were twisted and connected to the stimulator. By that, the stray field of the wire loop coarsely mimicked that of the cables in the axial imaging slice in the upper part of the head.

In order to correct for the effects of the stray field, we reconstructed the wire path from the PETRA images, calculated the wire-induced field using the Biot-Savart Law, and subtracted it from the measured $\Delta \mathrm{B}_{\mathrm{z}, \mathrm{c}}$ image. We validated this correction method by comparing the corrected $\Delta \mathrm{B}_{\mathrm{z}, \mathrm{c}}$ images with the results of control measurements without current injection. Histograms of both measurements were obtained, and the mean and standard deviation of Gaussian distributions fitted to the histograms were compared. For both the experiments with and without current flow, $\mathrm{N}_{\text {meas }}=24$ measurements were used. The experiments were repeated twice to test the reproducibility of the results.

\section{Experiment 4: Dependence of measurement efficiency on repetition time}

In our prior study (Göksu et al., 2017), we employed phantom experiments and simulations to demonstrate a strong influence of the SSFP-FID repetition time $T_{R}$ (Fig. 1b) on the efficiency of the MRCDI measurements. We derived an optimal value of $T_{R}=120 \mathrm{~ms}$, which is higher than usually employed in order to allow for sufficient phase accumulation. However, a long $T_{R}$ can also increase the influence of physiological noise on the measurements, leading us to re-evaluate the impact of $T_{R}$ on measurement efficiency in the in-vivo case.

We performed SSFP-FID experiments in six participants, employing an R-L electrode montage. The experiments were repeated with and without current injection. The data of one subject was discarded due to severe motion artefacts. In each participant, three repetition times $T_{R}=[40,80,120]$ ms were tested in a random order. The number of measurement repetitions $\mathrm{N}_{\text {meas }}$ was adjusted to keep the total acquisition time $\mathrm{T}_{\text {tot }}$ close to 9 mins. The remaining MR sequence parameters were adjusted to optimize the measurement sensitivity and image quality for the given $T_{R}$ : 
297

298

299

300

301

302

303

304

305

306

307

308

309

310

311

312

313

314

315

316

317

318

319

320

321

322

323

324

325

326

327

328

329

- $\mathrm{T}_{\mathrm{R}}=40 \mathrm{~ms}: \mathrm{T}_{\mathrm{GE}}=20 \mathrm{~ms}, \mathrm{~N}_{\mathrm{GE}}=1, \mathrm{BW}=276 \mathrm{~Hz} /$ pixel, $\mathrm{N}_{\text {meas }}=72$

- $\mathrm{T}_{\mathrm{R}}=80 \mathrm{~ms}: \mathrm{T}_{\mathrm{GE}}=[7.46,19.73,31.86,43.99,56.13] \mathrm{ms}, \mathrm{N}_{\mathrm{GE}}=5, \mathrm{BW}=88 \mathrm{~Hz} /$ pixel, $\mathrm{N}_{\text {meas }}=36$

- $\mathrm{T}_{\mathrm{R}}=120 \mathrm{~ms}: \mathrm{T}_{\mathrm{GE}}=[8.33,22.43,36.53,50.63,64.73,79.00,93.16] \mathrm{ms}, \mathrm{N}_{\mathrm{GE}}=7, \mathrm{BW}=75$ $\mathrm{Hz} /$ pixel, $\mathrm{N}_{\text {meas }}=24$

The $\Delta \mathrm{B}_{z, \mathrm{c}}$ images were corrected for the cable-induced stray fields as described above. Histograms of the $\Delta \mathrm{B}_{z, \mathrm{c}}$ images without current injection were obtained, and the mean and standard deviation of Gaussian distributions fitted to the histograms were determined.

\section{Experiment 5: $\Delta B_{z, c}$ measurements for two different electrode montages}

We compared the $\Delta \mathrm{B}_{z, \mathrm{c}}$ images obtained for R-L versus A-P electrode montages in six participants. The sequence parameters were $\mathrm{T}_{\mathrm{R}}=120 \mathrm{~ms}, \mathrm{~N}_{\text {meas }}=24\left(\mathrm{~T}_{\mathrm{tot}} \approx 9\right.$ mins $)$ and $\mathrm{N}_{\mathrm{GE}}=7$. The measurements were repeated with and without current injection. The data of one subject was discarded due to motion artefacts. The $\Delta \mathrm{B}_{\mathrm{z}, \mathrm{c}}$ images were corrected for the cable-induced stray fields. Histograms of the $\Delta \mathrm{B}_{\mathrm{z}, \mathrm{c}}$ images without current injection were obtained, and the mean and standard deviation of Gaussian distributions fitted to the histograms were determined.

\section{FEM simulations of the current flow and the induced magnetic field}

We compared the $\Delta \mathrm{B}_{z, \mathrm{c}}$ images measured in experiment 5 with simulated images, using FEM calculations of the current flow distribution inside the head based on our open-source pipeline SimNIBS 2 (www.simnibs.org; A Thielscher et al., 2015). An anatomically realistic volume conductor model was automatically created from the structural $\mathrm{T}_{1}$ and $\mathrm{T}_{2}$-weighted MR images. The model consists of five tissue compartments, namely brain gray matter (GM), white matter (WM), cerebrospinal fluid (CSF), skull and scalp. Isotropic ohmic conductivities were assigned to the tissues (WM: 0.126 S/m, GM: 0.275 S/m, CSF: 1.654 S/m, bone: 0.010 S/m, scalp: 0.465 S/m) (Thielscher et al., 2011). The electrode positions were determined from the PETRA images. The electrode pads were modelled as disks with $50 \mathrm{~mm}$ diameter and $5 \mathrm{~mm}$ thickness with a conductivity of $1.0 \mathrm{~S} / \mathrm{m}$. For the FEM calculations, Dirichlet boundary conditions for the electrostatic potential were applied at the electrode surfaces (Saturnino et al., 2015). The simulations were performed for both R-L and A-P montages, assuming a current strength of $I_{c}=1 \mathrm{~mA}$. The Biot-Savart Law was applied to the calculated current density distribution $\vec{J}$ in order to determine the $\Delta \mathrm{B}_{\mathrm{z}, \mathrm{c}}$ image.

\section{Reconstruction of current density images}

The measured $\Delta \mathrm{B}_{z, \mathrm{c}}$ images for the two electrode montages R-L and A-P (experiment 5) were used to determine current density distributions. We reconstructed the $\mathrm{x}$ - and $\mathrm{y}$-component of the current density in the imaging slice using the approach explained in (Ider et al., 2010; Park et al., 2007). The recovered current density $\vec{J}_{\text {rec }}$, termed "projected current density" in (Park et al., 2007), is given as 


$$
\vec{J}_{r e c}=\vec{J}_{0}+\frac{1}{\mu_{0}}\left(\frac{\partial\left(\Delta \mathrm{B}_{\mathrm{z}, \mathrm{c}}-\Delta \mathrm{B}_{\mathrm{z}, \mathrm{c}}^{0}\right)}{\partial y},-\frac{\partial\left(\Delta \mathrm{B}_{\mathrm{z}, \mathrm{c}}-\Delta \mathrm{B}_{\mathrm{z}, \mathrm{c}}^{0}\right)}{\partial x}, 0\right)
$$

with $\mu_{0}$ being the permeability of free space. The variables $\vec{J}_{0}$ and $\Delta \mathrm{B}_{\mathrm{z,c}}^{0}$ denote the current density and magnetic field distributions that would occur for a uniform conductivity distribution inside the head. They were determined using FEM calculations. The projected current density images were reconstructed from both the measurements with and without stray field correction, and compared with the simulation results. A median filter ( $3 \times 3$ neighborhood) was applied to the $\Delta \mathrm{B}_{\mathrm{z}, \mathrm{c}}$ measurements to remove spatial high-frequency noise before applying the reconstruction algorithm. For comparability, the same filter was applied to the simulated $\Delta \mathrm{B}_{\mathrm{z}, \mathrm{c}}$, even though it affected the images only marginally. There are more advanced filtering (Lee et al., 2011) and current density reconstruction (Ider et al., 2010; Park et al., 2007) techniques, which might perform slightly better. However, they are beyond the scope of this study.

\section{RESULTS}

\section{Subject Experiences}

For both the MESE and SSFP-FID methods, the synchronized injected current induced similar side effects in each of the subjects. All subjects reported phosphenes, which were stronger for the A-P compared the R-L electrode configuration. They also experienced subtle tingling near the electrodes, which disappeared after a short while. None of the subjects reported any discomfort due to the current injection.

\section{Experiment 1: Single-vs. Multi-gradient-echo acquisition}

For both the MESE and SSFP-FID measurements, the evaluation of the $\Delta \mathrm{B}_{z, \mathrm{c}}$ images acquired without current injection (Fig. 2a) shows that the multi-gradient-echo readouts consistently reduce the noise floor. In case of multiple echoes, the depicted $\Delta \mathrm{B}_{\mathrm{z}, \mathrm{c}}$ images are the weighted sum of the single echo results, with the weighting factors being proportional to the inverse of the variances of the images (Göksu et al., 2017). The better quality of the multi-gradient-echo results is corroborated by the lower mean values and standard deviations obtained for the $\Delta \mathrm{B}_{z, \mathrm{c}}$ images of the multi-gradient-echo readouts, as listed in Table 1 (Supplementary Fig. S1 shows the corresponding histograms). In this respect, the mean values indicate $\Delta \mathrm{B}_{z, \mathrm{c}}$, offsets, while the standard deviations characterize the "noise power", i.e. the strength of the spatial fluctuations of the noise. As a side note, the MESE measurements with multi-gradient-echo readouts have the lowest noise standard deviations across all 
four tested conditions. However, it should be noted that the listed values are not normalized per unit time, and the total scan time of MESE was two times longer than that of the SSFP-FID counterparts. The use of multi-gradient-echo readouts also helps to improve the quality of the MR magnitude images (Supplementary Fig. S2) and $\Delta \mathrm{B}_{z, c}$ images obtained with current injection (Fig. 2b). Specifically, the results for the single-gradient-echo readouts suffer from ghosting-like patterns, which are absent when multi-gradient-echo readouts are used. Visual inspection suggests further that the multi-gradient-echo readouts result in more similar $\Delta \mathrm{B}_{\mathrm{z}, \mathrm{c}}$ images for the MESE and SSFP-FID measurements in each of the three subjects.

\section{Experiment 2: Linear dependence of the measured $\Delta B_{z, c}$ on current strength}

Figure 3a shows the MR magnitude and $\Delta \mathrm{B}_{z, \mathrm{c}}$ images for MESE and SSFP-FID measurements performed at $I_{c}=1 \mathrm{~mA}$. In each of the subjects, the $\Delta B_{z, c}$ images of the MESE and SSFP-FID measurements show a good similarity. Average $\Delta \mathrm{B}_{z, \mathrm{c}}$ values were extracted from the indicated ROIs for each of the four tested current strengths and plotted against the current strength in Fig. 3b. In all cases, the fitted regression models are highly significant, demonstrating a good linear dependency (Table 2). The mean shifts $\beta_{0}$ (i.e., the intercepts of the fits) are close to zero in all cases, which proves the absence of systematic biases. For all three subjects, the slopes $\beta_{1}$ are similar between the MESE and SSFP-FID results. For SSFP-FID, the small standard errors indicate a good accuracy of the $\Delta B_{z, c}$ results that were obtained by averaging across twelve measurements.

\section{Experiment 3: Correction of cable-induced magnetic stray fields}

The $\Delta \mathrm{B}_{\mathrm{z}, \mathrm{c}}$ fields created by currents flowing in a wire loop around the head and measured using SSFPFID are shown in Fig. $4 \mathrm{~b}$. Corrected images were obtained by subtracting $\Delta \mathrm{B}_{\mathrm{z}, \mathrm{c}}$ fields that were determined via forward calculations based on the reconstructed wire paths and the Biot-Savart Law (Fig. 4c).

Comparing the corrected images with control $\Delta \mathrm{B}_{\mathrm{z}, \mathrm{c}}$ measurements without current injection (Fig. $4 \mathrm{~d}$ ) demonstrates that the remaining noise after the correction is in a similar range to that of the control images. This is confirmed by evaluating the mean values and standard deviations of the $\Delta \mathrm{B}_{z, \mathrm{c}}$ images, as listed in Table 3. For both experimental runs, the mean values of the corrected and control results are close to zero. The standard deviations are slightly higher for the corrected results, indicating a small residual effect that was not corrected by the subtraction procedure. The underlying reason might be small inaccuracies in determining the wire paths from the PETRA images.

\section{Experiment 4: Dependence of SSFP-FID measurement efficiency on repetition time}

The $\Delta \mathrm{B}_{z, \mathrm{c}}$ images acquired using SSFP-FID at three different repetition times, both with and without current injection, are shown in Figure 5. The images with current injection were corrected for the impact of the cable-induced stray fields as described above. The SNR of the images acquired at $T_{R}=$ 
$40 \mathrm{~ms}$ is clearly lower than obtained at the two other repetition times. The results obtained at $\mathrm{T}_{\mathrm{R}}=80$ $\mathrm{ms}$ and $\mathrm{T}_{\mathrm{R}}=120 \mathrm{~ms}$ exhibit similar sensitivities to the current-induced magnetic field changes. An exception is the $\Delta \mathrm{B}_{z, \mathrm{c}}$ image obtained for subject $\mathrm{S}_{4}$ without current injection (fourth row of Fig. 5a), which has a poor quality compared to the other results, presumably due to motion outside of the imaged slice. This occurred even though the MR magnitude images show no considerable image artifacts (Supplementary Fig. S3). Comparison of the standard deviations of the $\Delta \mathrm{B}_{\mathrm{z}, \mathrm{c}}$ images obtained without current injection confirms the visual impression (Table 4). On average, the standard deviation is reduced by $61 \%$ for $T_{R}=80 \mathrm{~ms}$ and $53 \%$ for $T_{R}=120 \mathrm{~ms}$ compared to the measurements at $T_{R}=$ $40 \mathrm{~ms}$. The measurements using $T_{R}=80 \mathrm{~ms}$ perform slightly better than those with $T_{R}=120 \mathrm{~ms}$ in four out of five subjects.

\section{Experiment 5: $\Delta B_{z, c}$ measurements for two different electrode montages}

The $\Delta \mathrm{B}_{\mathrm{z}, \mathrm{c}}$ images obtained by SSFP-FID measurements for the R-L and A-P electrode montages are shown in Figure 6, both without (Fig. 6b) and with correction of the cable-induced stray fields (Fig. $6 \mathrm{c}$ ). Visual comparison confirms the importance of applying the correction (please note, that the bluered patterns are actually inversed between uncorrected and corrected images). Focusing on the corrected images (Fig. 6c), the results of the A-P montage exhibit very similar spatial distributions of the current-induced magnetic fields across the five subjects, while the obtained peak intensities clearly vary. The results obtained with the R-L montage differ more between subjects, with the variation in the electrode positions likely contributing to these differences.

The control $\Delta \mathrm{B}_{z, \mathrm{c}}$ images obtained without current injection (Supplementary Fig. S4) exhibit an average mean shift of $\mu_{\triangle \mathrm{Bz}, \mathrm{c}}=0.005 \mathrm{nT}$ (averaged across the five subjects) and an average standard deviation of $\sigma_{\Delta \mathrm{Bz}, \mathrm{c}}=0.111 \mathrm{nT}$. These values ensure a sufficient sensitivity when measuring magnetic field changes caused by the current flow at the chosen strength of $1 \mathrm{~mA}$.

\section{Comparison of measured and simulated current-induced magnetic fields $\Delta B_{z, c}$}

We simulated the current-induced magnetic field for both the R-L and A-P electrode montages (Fig. 7 a shows exemplarily the results for subject $S_{1}$ ). In general, the simulated and measured fields exhibit similar spatial distributions and variations, supporting the validity of the measurements. Scatter plots of the measurements (with and without correction of the stray fields) versus simulations show clear linear dependencies for the corrected $\Delta \mathrm{B}_{\mathrm{z}, \mathrm{c}}$ data, which are absent for the uncorrected measurements (Fig. $7 \mathrm{~b}$ depicts the results for $\mathrm{S}_{1}$ ). Correspondingly, fitting linear regression models to the dependencies between corrected $\Delta \mathrm{B}_{\mathrm{z}, \mathrm{c}}$ measurements and simulations reveals significant results for all subjects (Table 5), with the coefficients of determination being on average 0.68 and 0.88 for the R-L and A-P montages. Interestingly, the estimated slopes are slightly lower than unity. That is, the simulations underestimate $\Delta \mathrm{B}_{\mathrm{z}, \mathrm{c}}$ slightly, but quite systematically in 9 out of the 10 measurements. It 
is worth noting that we do not expect identical results, as the simulations were based on a head model that employed standard conductivity values from literature.

\section{Comparison of the current density measurements and simulations}

We reconstructed the $\mathrm{x}$ - and $\mathrm{y}$-components of the current density distribution in the imaging slice from the $\Delta \mathrm{B}_{z, c}$ measurements (with and without stray field correction) and additionally from the simulated $\Delta \mathrm{B}_{z, \mathrm{c}}$ data in the five subjects for both R-L and A-P electrode montages. The results of the first subject are exemplarily shown in Figure 8a (Supplementary Fig. S5 lists the results of the other subjects). For the simulations, the reconstructed current densities $\vec{J}_{\text {rec }}$ differ markedly from the original current densities $\vec{J}_{\text {FEM }}$ that were determined via FEM calculations and served to calculate the $\Delta \mathrm{B}_{\mathrm{z}, \mathrm{c}}$ distributions via the Biot-Savart Law. While coarse features of the current flow pattern such as generally higher current densities close to the electrodes and in the longitudinal fissure (for the A-P montage) are maintained, fine inflow effects in the sulci are mostly lost. Visual comparison of the current density reconstructions from the uncorrected versus corrected $\Delta \mathrm{B}_{\mathrm{z,c}}$ measurements reveals that the current densities close to the electrodes are overestimated when the $\Delta \mathrm{B}_{z, \mathrm{c}}$ data is not corrected for the cable-induced stray fields. In addition, increased current densities in the CSF-filled longitudinal fissure are only observable for the corrected case. Comparing the current density distributions reconstructed from the measurements versus the simulations by means of scatter plots (Fig. 8b) and linear regression analyses (Tables 6 and 7) confirms that the difference between measurements and simulations is overestimated without stray field correction. Specifically, the slopes of the regression lines increase by on average 0.16 (uncorrected vs. corrected: 0.65 vs. 0.81 , pooled across A-P and RL). Also for the corrected data, the slopes are still lower than unity, i.e. the simulations systematically underestimate the current densities by on average $24 \%$. The coefficients of determination are only slightly increased for the corrected data, for which they reach on average 0.71 .

\section{DISCUSSION AND CONCLUSIONS}

We tested two MR sequences, MESE and SSFP-FID, for measurements of weak current-induced magnetic fields in the human brain. The sequences were previously optimized using extensive computer simulations and phantom tests (Göksu et al., 2017) to maximize their sensitivity to the current-induced fields. Here, we assessed their performance in-vivo and demonstrated that both sequence types could be successfully used to reveal the magnetic field distributions for a current strength of $1 \mathrm{~mA}$, in turn allowing us to reconstruct the current flow distribution in the brain.

\section{Optimization and validation of the MR sequences and measurement procedures}


Our results demonstrated the need to adapt the employed sequences for in-vivo application by including multi-gradient-echo acquisitions. Specifically, long echo times (MESE) and repetitions times (SSFP-FID) are required to maximize the sensitivity of the measurements to the current-induced magnetic fields (Göksu et al., 2017). This in turn decreases their robustness to physiological noise (e.g., due to respiration, blood flow and small subject movement) when single-echo readouts with low

465

466

467

468

469

470

471

472

473

474

475

476

477

478

479

480

481

482

483

484

485

486

487

488

489

490

491

492

493

494

495

496 bandwidths are used. Our results show that multi-gradient-echo readouts at higher bandwidths improve the image quality and allow selecting long echo and repetition times to maximize sensitivity, even though the total available readout period is slightly shortened by the time needed for the additional gradient switching in that case.

In contrast to the better efficiency of SSFP-FID compared to MESE observed in the prior phantom tests, both sequence types had similar noise levels in the in-vivo case when matching the total acquisition time. Specifically, comparing the average noise standard deviations listed for MESE $\left(\mathrm{N}_{\mathrm{GE}}=5\right)$ in Table 1 to the results for SSFP-FID with $\mathrm{T}_{\mathrm{R}}=120 \mathrm{~ms}$ in Table 4 (both acquired with $\mathrm{T}_{\text {tot }} \approx$ 9 mins) reveals similar values. This indicates that physiological noise is a dominant factor that limits the sensitivity of the in-vivo measurements. In practice, the higher number of measurements that are obtained during SSFP-FID acquisitions open up a possibility of discarding (partial) measurements with strong noise, thereby possibly improving the quality of the final averaged magnetic field image. Nevertheless, MESE may still outperform SSFP-FID in multi-slice acquisition, as it allows for interleaved slice excitation without prolonging the total acquisition time (Göksu et al., 2017). The impact of physiological noise also became apparent when testing different repetition times for the SSFP-FID measurements. As expected, increasing $\mathrm{T}_{\mathrm{R}}$ from $40 \mathrm{~ms}$ to $80 \mathrm{~ms}$ increased the measurement sensitivity. However, an additional increase to $120 \mathrm{~ms}$ tended to decrease the sensitivity of the in-vivo results slightly again, in contrast to the theoretical and phantom results. This indicates that a $T_{R}$ moderately below the theoretically optimal value can be chosen for in-vivo applications without losing sensitivity, while potentially improving robustness.

For both sequence types, the dependence of the measured magnetic field on the current strength exhibited a good linearity. In each of the three tested subjects, the slopes of the linear fits of the results obtained with the two sequences were similar. This validates the chosen scaling factor $\mathrm{m}_{\text {seq }}$ for the SSFP-FID measurements (Eq. 2), which relates the magnetic field and phase changes, and which was determined via spin simulations (Göksu et al., 2017).

We have demonstrated strong effects of the magnetic stray fields created by the current flow in the cables on the measured magnetic field and on the reconstructed current flow distributions, and have validated a correction method that employs delineations of the cable paths derived from structural images for forward calculations of the stray fields. While improved cable designs might help to ameliorate this problem, we would like to emphasize that even a small deviation from an ideal path parallel to the field direction of the scanner will cause non-negligible distortions of the measured field distributions when it occurs close to the measurement volume, e.g., $10 \mathrm{~cm}$ away. This effect results in 
497

498

499

500

501

502

503

504

505

506

507

508

509

510

511

512

513

514

515

516

517

518

519

520

521

522

523

524

525

526

527

528

529

530

531

532

533

miscalculated current flow distributions, and highlights the importance of controlling for and, if required, correcting the impact of the stray fields.

\section{Comparison of measured and simulated fields}

The measured magnetic fields showed a good correspondence to the fields obtained via FEM simulations, with average coefficient of determinations $\mathrm{R}^{2}$ of $68 \%$ and $88 \%$ for R-L and A-P montages. Following up on the reasons why the A-P montage is on average revealing a better correspondence might be interesting for future studies. The simulations based on "standard" tissue conductivities taken from literature systematically underestimated the strength of the current-induced $\Delta B_{z, c}$ in 9 out of the 10 measurements (average regression slopes of 0.80 and 0.90 for R-L and A-P). Also the current density distributions estimated from the corrected magnetic field measurements and the FEM simulations were in good agreement, with an average coefficient of determination of $\mathrm{R}^{2}=71 \%$, with little difference between the R-L and A-P montages. The simulations underestimated the current strength on average by $24 \%$. The likely main reason are inaccurate ohmic conductivities assigned to the brain tissues in the FEM simulations. In addition, the use of isotropic conductivity values for white matter might affect the accuracy of the simulations, as its conductivity is known to be anisotropic (e.g., Nicholson, 1965). Future studies could therefore test whether the use of conductivity tensors estimated from diffusion MRI (e.g., Opitz et al., 2011) improves the fit between measurements and simulations. Along similar lines, it could be tested whether more detailed models of the pad electrodes (Saturnino et al., 2015) increase the fit. Interestingly, recent studies using invasive in-vivo recordings to measure the electric field injected by transcranial weak current stimulation indicate that FEM simulations based on standard conductivity values similar to the ones used here over- rather than underestimate the electric field strength (Huang et al., 2017; Opitz et al., 2016). This apparent contradiction might be resolved by considering that we reconstructed the current density rather than the electric field. Huang et al. (2017) derived individually optimized ohmic conductivities to best fit the simulated to the measured electric fields, and found that optimization resulted in higher-thanstandard tissue conductivities consistently across subjects. Increasing the conductivity of brain tissue would in turn tend to increase the current strength inside the skull, in line with our results. While this explanation seems plausible, it should be followed up, e.g. by future simulation work.

\section{Prior Studies}

To our knowledge, only two prior studies report in-vivo MR measurements of current-induced magnetic fields in the human brain. In (Jog et al., 2016), standard field mapping sequences were employed to measure the constant fields of direct currents. While the use of standard sequences has the advantage that $3 \mathrm{D}$ coverage can be readily achieved, this approach is not robust to slow temporal drifts of the MR signal that occur due to both technical and physiological reasons, inherently limiting the achievable sensitivity. The results presented in (Kasinadhuni et al., 2017) were based on a 
measurement approach that was more similar to the approach tested here. However, their method is comparably less sensitive to current-induced field changes and the results were not corrected for cable-induced stray fields. The spatial patterns of the measured magnetic field distributions reported in that study vary substantially across subjects, despite using the same electrode locations. The peak magnetic field values exceed those, which we obtained for the uncorrected images, and are consistently higher than those indicated by their and our FEM simulations. Even when considering that a higher current strength of $1.5 \mathrm{~mA}$ was applied, these observations indicate that cable-induced stray fields likely affected the results of that study. As the employed current flow reconstruction algorithm was based on first order spatial derivatives of the measured current-induced magnetic field (Ider et al., 2010; Park et al., 2007), any non-constant stray field will distort the reconstructed current flow. This opens the possibility that also the substantial differences between the measured and simulated current density reconstructions reported in (Kasinadhuni et al., 2017) might have been amplified by neglecting putative cable-induced stray fields. As a side note, the detrimental effects of cable-induced stray fields on the reconstructed current flow do not occur for methods which rely on the Laplacian of $\Delta \mathrm{B}_{z, \mathrm{c}}$ (e.g., Ider et al., 2010), as the Laplacian of the cable-induced stray magnetic fields is zero inside the imaging region. However, as these methods employ second derivatives, they might suffer more from amplified noise in the reconstructed current density images.

\section{Limitations and Future Work}

The main focus of our study was on the optimization and validation of the MR sequences and measurement protocol. In the future, the measurement sensitivity can possibly be further increased by using pulse sequences such as balanced alternating steady-state free precession (bSSFP) which exhibits a more than 10 times higher phase sensitivity (Bieri et al., 2006; Minhas et al., 2010). A higher sensitivity would be beneficial to limit scan time when aiming to extend the spatial coverage towards multiple slices. Increasing the current strength from $1 \mathrm{~mA}$ up to $2 \mathrm{~mA}$ is also feasible, but requires careful piloting. Stronger currents also increase the side effects such as tickling and pain sensations underneath the electrodes, which makes the measurements less comfortable for the participants and might result in stronger head movement. In addition, the correction method for the cable-induced stray field used here requires manual tracking of the cable paths that are, however, well visible as dark regions inside the bright Play-Doh. The tracking accuracy depends on the spatial resolution of the employed PETRA images $(0.9 \mathrm{~mm}$ iso-voxel in our experiments, which is sufficiently small to guarantee sufficient accuracy). It would be desirable to optimize the cable design in order to reduce the influence of the cable-induced stray fields, which might help to further increase the robustness of the final results.

The employed current density reconstruction can be further optimized. The observed similarity between the reconstructions from measured and simulated data suggests that the method in its current form is already sufficient to obtain a coarse approximation of the current flow. Replacing the median 
571 filter used to denoise the $\Delta \mathrm{B}_{\mathrm{z}, \mathrm{c}}$ image before applying the reconstruction algorithm by more advanced

572 filter approaches (Lee et al., 2011) might help to reveal some more detail in the reconstructed current

573 density images. The reconstruction was based on the simplifying assumption that the $\mathrm{x}-$ and $\mathrm{y}$ -

574 components of the current-induced magnetic field are small and can be neglected (Sajib et al., 2012).

575 This also implies that the measured conductivity distribution does not vary along the z-direction.

576 However, the head and brain clearly vary along $\mathrm{z}$, so that accurate estimations of the current flow

577 require assumptions that are more realistic. Combined with the imaging of multiple slices,

578 reconstructing the current flow from $3 \mathrm{D} \Delta \mathrm{B}_{\mathrm{z,c}}$ data should help to increase the accuracy of the

579 reconstruction (Ider et al., 2010). However, it should be noted that these limitations do not affect our

580 finding that the FEM simulations underestimated the current strength, as we applied the same

581 reconstruction steps to the simulation results rather than using the originally simulated current

582 distribution for comparison (Fig. 8a). Finally, it will be interesting to explore the usage of the

583 measured $\Delta \mathrm{B}_{\mathrm{z}, \mathrm{c}}$ data for the estimation of individual tissue conductivities (Kwon et al., 2016).

584

585 Conclusion

586 We have demonstrated the feasibility of reliable MRCDI measurements in-vivo in the human brain at

587 a current strength of $1 \mathrm{~mA}$. Future studies might aim to further improve the sensitivity of the MR

588 methods and their robustness to physiological noise, as well as to extend their spatial coverage

589 towards multiple slices. Our results are promising and indicate that MRCDI measurements combined

590 with the reconstruction of current densities and tissue conductivities (Eyüboğlu, 2006c; Ider et al.,

591 2010; Park et al., 2007; Seo et al., 2003; Seo and Woo, 2011) might be useful for validating

592 simulations based on volume conductor models of the head and for improving the accuracy of the

593 simulations.

594

595

596 ACKNOWLEDGEMENTS

597 This study was supported by the Lundbeck foundation (grant R118-A11308 to AT and grant R59

598 A5399 to HR).

599 
Figure 1. Schematic diagrams of the MESE and SSFP-FID sequences (please see Göksu et al., 2017 for details). (a) Diagram of the MESE sequence. The sequence is composed of a $90^{\circ}$ excitation pulse preceding repetitive $180^{\circ}$ refocusing pulses, so that multiple spin echoes are created. The injected bipolar current waveform is synchronized with the radio frequency (RF) pulses, so that the phase of the continuous complex transverse magnetization $(\Varangle \mu)$ increases linearly over time. The measurement is performed twice with opposite current injection profiles (indicated by red and green dashed lines), and the difference between the phase images is used to determine the current-induced magnetic field. Either single-gradient-echo readouts (single $\mathrm{G}_{\mathrm{r}}$ ) or multi-gradient-echo readouts with fly-back (multi $\mathrm{G}_{\mathrm{r}}$ ) are used. (b) Diagram of the SSFP-FID sequence. The sequence is composed of repetitive inphase excitation pulses with constant tip angle and constant repetition time $T_{R}$. A bipolar current waveform is injected in synchrony with the SSFP-FID sequence. The current-induced phase of the continuous complex transverse magnetization evolves in opposite directions in odd and even $T_{R}$ periods (indicated by red and green lines), which results in two different steady-state conditions with opposite phases. Either single-gradient-echo readouts (single $\mathrm{G}_{\mathrm{r}}$ ) or multi-gradient-echo readouts with fly-back (multi $\mathrm{G}_{\mathrm{r}}$ ) are used.

Figure 2. Experiment 1: Comparison of single- vs. multi-gradient-echo acquisition in three subjects. The $T_{E S}$ (MESE) and $T_{R}$ (SSFP-FID) times were kept identical between both cases. All images are shown in radiological convention, with the orientation indicated for the lower right slice in subfigure b. (a) $\Delta \mathrm{B}_{\mathrm{z}, \mathrm{c}}$ images of the measurements without current injection. For both MESE and SSFP-FID $\left(\mathrm{T}_{\mathrm{R}}=120 \mathrm{~ms}, \mathrm{~N}_{\text {meas }}=12\right)$, the results of the multi-gradient-echo acquisitions exhibit a lower noise floor than those of the single-gradient-echo acquisitions. (b) $\Delta \mathrm{B}_{\mathrm{z}, \mathrm{c}}$ images of the measurements with current injection. For better visualization of the spatial patterns, mean-corrected images are shown (i.e., the average $\Delta \mathrm{B}_{\mathrm{z}, \mathrm{c}}$ in the brain was subtracted). The quality of the images is improved by the use of multigradient-echo readouts, which prevent the ghosting-like patterns observed in the results of the singlegradient-echo acquisitions. Please note that the total acquisition times differed for MESE $\left(\mathrm{T}_{\text {tot }} \approx 9\right.$ mins) and SSFP-FID ( $\mathrm{T}_{\text {tot }} \approx 4.5 \mathrm{mins}$ ) in this experiment, as the primary goal was to compare singleversus multi-gradient-echo readouts.

Figure 3. Experiment 2: Test of the linear dependence of the measured $\Delta \mathrm{B}_{z, \mathrm{c}}$ on the applied current strength in three subjects, performed for both MESE and SSFP-FID $\left(T_{R}=120 \mathrm{~ms}, \mathrm{~N}_{\text {meas }}=12\right)$ with multi-gradient-echo readouts. (a) Magnitude and $\Delta \mathrm{B}_{z, \mathrm{c}}$ images for the measurements at $\mathrm{I}_{\mathrm{c}}=1 \mathrm{~mA}$.

634 The black rectangles depict the regions-of-interest (ROIs), in which the average $\Delta B_{z, c}$ was extracted. 635 In subject $S_{1}$, a line-like artifact is visible in the MESE $\Delta \mathrm{B}_{z, c}$ images in the phase encoding direction 
636

637

638

639

640

641

642

643

644

645

646

647

648

649

650

651

652

653

654

655

656

657

658

659

660

661

662

663

664

665

666

667

668

669

670

671

672

and to a lesser extent also in the SSFP-FID results. The artifact is consistent with flow effects from vessels. We did not observe this type of artifact again. (b) Dependency of the average $\Delta \mathrm{B}_{z, \mathrm{c}}$ in the ROI on the applied current strength. For MESE, the results of the two measurements are shown as blue and orange lines, and their average is shown as a green line. For SSFP-FID, the average of the 12 measurements is shown; the bars represent the standard error.

Figure 4. Experiment 3: Correction of the cable-induced magnetic stray fields for SSFP-FID measurements $\left(T_{R}=120 \mathrm{~ms}, \mathrm{~N}_{\text {meas }}=24\right)$ with multi-gradient-echo readouts in four subjects (no tissue current). The experiments were repeated twice, and the figure shows the results of the first experimental run. (a) Magnitude images. (b) Uncorrected $\Delta \mathrm{B}_{z, \mathrm{c}}$ images showing the stray field generated by the current flow in the wire loop around the head. (c) Corrected $\Delta \mathrm{B}_{\mathrm{z}, \mathrm{c}}$ images, in which the stray field was calculated based on the reconstructed wire path and subtracted from the measured $\Delta \mathrm{B}_{\mathrm{z}, \mathrm{c}}$. (d) $\Delta \mathrm{B}_{\mathrm{z}, \mathrm{c}}$ images of the control measurements performed without current injection.

Figure 5. Experiment 4: Comparison of SSFP-FID measurements with multi-gradient-echo readouts performed in five subjects using three different repetition times $T_{R}$. (a) $\Delta B_{z, c}$ images of the experiments performed without current injection. (b) $\Delta \mathrm{B}_{z, \mathrm{c}}$ images of the experiments with current injection. The experiments using $\mathrm{T}_{\mathrm{R}}=40 \mathrm{~ms}$ exhibit the highest noise levels. The total acquisition time was kept the same for the three repetition times by adapting the number of measurements $\left(\mathrm{T}_{\mathrm{R}}=40\right.$ ms: $\mathrm{N}_{\text {meas }}=72 ; \mathrm{T}_{\mathrm{R}}=80 \mathrm{~ms}: \mathrm{N}_{\text {meas }}=36 ; \mathrm{T}_{\mathrm{R}}=120 \mathrm{~ms}: \mathrm{N}_{\text {meas }}=24$ ).

Figure 6. Experiment 5: SSFP-FID measurements $\left(T_{R}=120 \mathrm{~ms}, \mathrm{~N}_{\text {meas }}=24\right)$ with multi-gradient-echo readouts of five subjects for the R-L and A-P electrode montages. (a) Magnitude images. (b) Uncorrected $\Delta \mathrm{B}_{\mathrm{z,c}}$ images (left column: R-L montage; right column: A-P montage). (c) Corrected $\Delta \mathrm{B}_{\mathrm{z}, \mathrm{c}}$ images. The electrode positions are indicated as black boxes. Note that cable contributions dominate the uncorrected images.

Figure 7. Experiment 5: (a) SSFP-FID measurements and FEM simulations of the current-induced $\Delta B_{z, c}$ for subject $S 1$ in Fig. 6 . Shown are the MR magnitude image, and the corrected $\Delta B_{z, c}$ images and FEM results for the both the R-L and A-P montages. The electrode positions are shown as black rectangles. (b) Scatter plots of the $\Delta \mathrm{B}_{\mathrm{z}, \mathrm{c}}$ measurements versus simulations (left column: R-L montage; right column: A-P montage). The results are plotted for the cases with (blue) and without (orange) cable-induced $\Delta \mathrm{B}_{z, \mathrm{c}}$ correction. The results without stray field correction have no correspondence to the simulations. The red regression lines are based on a linear regression of the corrected $\Delta \mathrm{B}_{z, \mathrm{c}}$ measurement results versus the simulations (please refer to Table 5 for the results of the regression analyses for all five subjects). 
673 Figure 8. Experiment 5: (a) Reconstruction of the current density distributions from simulated and

674 measured $\Delta B_{z, c}$ images. The results for subject $S_{1}$ are exemplarily shown (upper row: R-L montage;

675 second row: A-P montage). The norm of the 2D current densities is depicted. Visual comparison of 676 the simulated current density distributions $\overrightarrow{\mathbf{J}}_{\text {FEM }}$ with their corresponding $\overrightarrow{\mathbf{J}}_{\text {rec }}$ images that were 677 reconstructed from the simulated $\Delta \mathrm{B}_{z, \mathrm{c}}$ images shows that the reconstruction algorithm recovers only 678 the coarse features of the current flow pattern. Specifically, higher current densities close to the 679 electrodes and in the longitudinal fissure are maintained. Visual comparison of the reconstructions 680 from uncorrected and corrected $\Delta \mathrm{B}_{\mathrm{z}, \mathrm{c}}$ measurements reveals that the reconstructions from the 681 uncorrected measurements overestimate the current densities close to the electrodes. For the A-P 682 montage, an increased current flow in the longitudinal fissure is only visible for the corrected 683 measurements. (b) Scatter plots of the projected current flow measurements versus simulations for 684 subject $S_{1}\left(1^{\text {st }}\right.$ row: R-L montage; $2^{\text {nd }}$ row: A-P montage). The results are plotted for the cases with 685 (blue) and without (red) cable-induced stray magnetic field correction. The results without stray field 686 correction overestimate the current flow density, resulting in a smaller slope of the fitted regression 687 line (please refer to Table 6 for the results of the regression analyses for all five subjects).

688

689

690 


\begin{tabular}{|c|c|c|c|c|}
\cline { 2 - 5 } \multicolumn{1}{c|}{} & \multicolumn{2}{c|}{ MESE } & \multicolumn{2}{c|}{ SSFP-FID } \\
\cline { 2 - 5 } & $\begin{array}{c}\mathbf{N}_{\mathrm{GE}}=\mathbf{1} \\
\mu_{\Delta \mathrm{BZ}, \mathrm{c}}\left(\sigma_{\Delta \mathrm{BZ}, \mathrm{c}}\right)\end{array}$ & $\mathbf{N}_{\mathbf{G E}=\mathbf{5}}$ & $\mathbf{N}_{\mathbf{G E}}=\mathbf{1}$ & $\mathbf{N}_{\mathbf{G E}}=\mathbf{7}$ \\
\hline$S_{1}$ & $0.066(0.166)$ & $0.037(0.086)$ & $-0.043(0.149)$ & $0.026(0.123)$ \\
\hline$S_{2}$ & $0.126(0.186)$ & $0.089(0.117)$ & $-0.024(0.201)$ & $-0.012(0.111)$ \\
\hline$S_{3}$ & $0.144(0.150)$ & $-0.035(0.103)$ & $-0.078(0.150)$ & $-0.041(0.151)$ \\
\hline Avg & $\mathbf{0 . 1 1 2 ( 0 . 1 6 7 )}$ & $\mathbf{0 . 0 3 0}(\mathbf{0 . 1 0 2})$ & $\mathbf{- 0 . 0 4 8}(\mathbf{0 . 1 6 7})$ & $\mathbf{- 0 . 0 0 9}(\mathbf{0 . 1 2 8})$ \\
\hline
\end{tabular}

693 694 695 696 697 698 699 8

99

Table 1. Experiment 1: Comparison of single- vs. multi-gradient-echo acquisition for the case without current injection in three subjects. The table lists the mean shifts $\mu_{\Delta \mathrm{Bz}, \mathrm{c}}$ and standard deviations $\sigma_{\Delta \mathrm{Bz}, \mathrm{c}}$ (given in brackets) of the noise distributions of $\Delta \mathrm{B}_{z, \mathrm{c}}$ values in the brain. The last row lists the average $\mu_{\Delta \mathrm{Bz}, \mathrm{c}}$ and average $\sigma_{\Delta \mathrm{Bz}, \mathrm{c}}$ values across subjects. The units are in nT. For both MESE and SSFP-FID, the multi-gradient-echo acquisitions have lower mean shifts and standard deviations.

\begin{tabular}{|c|c|c|c|c|c|c|}
\hline & \multicolumn{3}{|c|}{ MESE } & \multicolumn{3}{|c|}{ SSFP-FID } \\
\hline & $\mathbf{F}_{1,6}(\mathbf{p})$ & $\begin{array}{c}\boldsymbol{\beta}_{\mathbf{0}} \\
\text { in }[\mathrm{nT}]\end{array}$ & $\begin{array}{c}\boldsymbol{\beta}_{\mathbf{1}} \text { in } \\
{[\mathrm{nT} / \mathrm{mA}]}\end{array}$ & $F_{1,46}(p)$ & $\begin{array}{c}\boldsymbol{\beta}_{\mathbf{0}} \\
\text { in }[\mathrm{nT}]\end{array}$ & $\begin{array}{c}\boldsymbol{\beta}_{\mathbf{1}} \text { in } \\
{[\mathrm{nT} / \mathrm{mA}]}\end{array}$ \\
\hline $\mathrm{S}_{1}$ & $\begin{array}{c}57 \\
\left(<0.3 \cdot 10^{-3}\right)\end{array}$ & $\begin{array}{l}-0.05 \\
(0.08)\end{array}$ & $\begin{array}{c}0.90 \\
(0.12)\end{array}$ & $\begin{array}{c}46 \\
\left(<10^{-6}\right)\end{array}$ & $\begin{array}{l}-0.07 \\
(0.07)\end{array}$ & $\begin{array}{c}0.80 \\
(0.12)\end{array}$ \\
\hline $\mathrm{S}_{2}$ & $\begin{array}{c}50 \\
\left(<0.4 \cdot 10^{-3}\right)\end{array}$ & $\begin{array}{c}0.07 \\
(0.09)\end{array}$ & $\begin{array}{c}1.03 \\
(0.15)\end{array}$ & $\begin{array}{c}27 \\
\left(<10^{-5}\right)\end{array}$ & $\begin{array}{c}0.02 \\
(0.13)\end{array}$ & $\begin{array}{c}1.09 \\
(0.21)\end{array}$ \\
\hline $\mathrm{S}_{3}$ & $\begin{array}{c}1527 \\
\left(<10^{-6}\right)\end{array}$ & $\begin{array}{l}-0.04 \\
(0.02)\end{array}$ & $\begin{array}{c}1.44 \\
(0.04)\end{array}$ & $\begin{array}{c}225 \\
\left(<10^{-6}\right)\end{array}$ & $\begin{array}{l}-0.01 \\
(0.06)\end{array}$ & $\begin{array}{c}1.42 \\
(0.10)\end{array}$ \\
\hline
\end{tabular}

Table 2. Experiment 2: Linear fits of the measured dependence of $\Delta \mathrm{B}_{\mathrm{z,c}}$ on the applied current strength. The table lists the F- and p-values, the intercepts $\beta_{0}$ and the slopes $\beta_{1}$ of the fitted linear regression models. The standard errors of $\beta_{0}$ and $\beta_{1}$ are given in brackets. 


\begin{tabular}{|c|c|c|c|c|}
\cline { 2 - 5 } & \multicolumn{2}{|c|}{$\mathbf{1}^{\text {st }}$ Experiment } & \multicolumn{2}{c|}{$\mathbf{2}^{\text {nd }}$ Experiment } \\
\cline { 2 - 5 } & $\begin{array}{c}\mathbf{I}_{\mathbf{c}}=\mathbf{0} \mathbf{~ m A} \\
\mu_{\Delta \mathrm{Bz}, \mathrm{c}}\left(\sigma_{\Delta \mathrm{Bz}, \mathrm{c}}\right)\end{array}$ & $\begin{array}{c}\mathbf{I}_{\mathbf{c}}=\mathbf{1} \mathbf{~ m A}, \\
\mathbf{c o r r e c t e d}\end{array}$ & $\mathbf{I}_{\mathbf{c}}=\mathbf{0} \mathbf{~ m A}$ & $\begin{array}{c}\mathbf{I}_{\mathbf{c}}=\mathbf{1} \mathbf{~ m A}, \\
\mathbf{c o r r e c t e d}\end{array}$ \\
\hline $\mathrm{S}_{1}$ & $0.013(0.136)$ & $0.049(0.138)$ & $0.016(0.095)$ & $-0.011(0.194)$ \\
\hline $\mathrm{S}_{2}$ & $-0.067(0.120)$ & $-0.012(0.148)$ & $-0.022(0.091)$ & $0.012(0.133)$ \\
\hline $\mathrm{S}_{3}$ & $-0.110(0.110)$ & $-0.169(0.130)$ & $0.011(0.108)$ & $-0.018(0.129)$ \\
\hline$S_{4}$ & $0.031(0.072)$ & $0.110(0.170)$ & $0.103(0.096)$ & $-0.080(0.125)$ \\
\hline $\mathbf{A v g}$ & $\mathbf{- 0 . 0 3 3 ( \mathbf { 0 . 1 1 0 } )}$ & $\mathbf{- 0 . 0 0 6 ( \mathbf { 0 . 1 4 7 } )}$ & $\mathbf{0 . 0 2 7}(\mathbf{0 . 0 9 8})$ & $\mathbf{- 0 . 0 2 4}(\mathbf{0 . 1 4 5})$ \\
\hline
\end{tabular}

705

Table 3. Experiment 3: Correction of the cable-induced magnetic stray field for SSFP-FID measurements in four subjects. The experiment was repeated twice. The table lists the mean shifts $\mu_{\Delta \mathrm{Bz}, \mathrm{c}}$ and standard deviations $\sigma_{\Delta \mathrm{Bz}, \mathrm{c}}$ (given in brackets) of the distribution of $\Delta \mathrm{B}_{\mathrm{z}, \mathrm{c}}$ in the brain. The last row lists the average $\mu_{\Delta \mathrm{Bz}, \mathrm{c}}$ and average $\sigma_{\Delta \mathrm{Bz}, \mathrm{c}}$ values across subjects. The units are in $\mathrm{nT}$. Correcting the stray field induced by the wire loop around the head results in noise distributions, which are similar to those of the control measurements without current injection.

\begin{tabular}{|c|c|c|c|}
\cline { 2 - 4 } \multicolumn{1}{c|}{} & $\begin{array}{c}\mathbf{T}_{\mathbf{R}}=\mathbf{4 0} \mathbf{~ \mathbf { S }} \\
\mu_{\Delta \mathrm{Bz}, \mathrm{c}}\left(\sigma_{\Delta \mathrm{Bz}, \mathrm{c}}\right)\end{array}$ & $\mathbf{T}_{\mathbf{R}}=\mathbf{8 0} \mathbf{~ \mathbf { ~ S }}$ & $\mathbf{T}_{\mathbf{R}}=\mathbf{1 2 0} \mathbf{~ \mathbf { S }}$ \\
\hline $\mathrm{S}_{1}$ & $0.039(0.202)$ & $0.115(0.092)$ & $-0.046(0.111)$ \\
\hline $\mathrm{S}_{2}$ & $-0.012(0.191)$ & $-0.007(0.073)$ & $-0.026(0.095)$ \\
\hline $\mathrm{S}_{3}$ & $-0.045(0.212)$ & $0.053(0.076)$ & $0.011(0.090)$ \\
\hline $\mathrm{S}_{4}$ & $-0.042(0.259)$ & $0.049(0.084)$ & $0.179(0.210)$ \\
\hline $\mathrm{S}_{5}$ & $-0.002(0.192)$ & $-0.052(0.091)$ & $0.038(0.084)$ \\
\hline Avg & $\mathbf{- 0 . 0 1 2 ( \mathbf { 0 . 2 1 1 } )}$ & $\mathbf{0 . 0 3 1}(\mathbf{0 . 0 8 3})$ & $\mathbf{0 . 0 3 1}(\mathbf{0 . 1 0 0})$ \\
\hline
\end{tabular}

712 Table 4. Experiment 4: Comparison of SSFP-FID measurements performed in five subjects without current injection for three different repetition times $T_{R}$. The table lists the mean shifts $\mu_{\Delta B z, c}$ and standard deviations $\sigma_{\Delta \mathrm{Bz}, \mathrm{c}}$ (given in brackets) of the noise distributions of $\Delta \mathrm{B}_{\mathrm{z}, \mathrm{c}}$ in the brain. The last row lists the average $\mu_{\Delta \mathrm{Bz}, \mathrm{c}}$ and average $\sigma_{\Delta \mathrm{Bz}, \mathrm{c}}$ values across subjects. The units are in nT. Both the 
716

717

719

\begin{tabular}{|c|c|c|c|c|c|c|}
\cline { 2 - 7 } \multicolumn{1}{c|}{} & \multicolumn{3}{|c|}{ R-L } & \multicolumn{2}{c|}{ A-P } \\
\cline { 2 - 7 } & $\begin{array}{c}\boldsymbol{\beta}_{\mathbf{0}} \\
\text { in [nT] }\end{array}$ & $\boldsymbol{\beta}_{\mathbf{1}}$ & $\mathbf{R}^{\mathbf{2}}$ & $\begin{array}{c}\boldsymbol{\beta}_{\mathbf{0}} \\
\text { in [nT] }\end{array}$ & $\boldsymbol{\beta}_{\mathbf{1}}$ & $\mathbf{R}^{\mathbf{2}}$ \\
\hline $\mathrm{S}_{1}$ & $\begin{array}{c}0.18 \\
\pm 0.002\end{array}$ & $\begin{array}{c}0.81 \\
\pm 0.12\end{array}$ & 0.87 & $\begin{array}{c}0.04 \\
\pm 0.003\end{array}$ & $\begin{array}{c}0.80 \\
\pm 0.004\end{array}$ & 0.91 \\
\hline $\mathrm{S}_{2}$ & $\begin{array}{c}0.04 \\
\pm 0.003\end{array}$ & $\begin{array}{c}0.75 \\
\pm 0.15\end{array}$ & 0.80 & $\begin{array}{c}0.06 \\
\pm 0.004\end{array}$ & $\begin{array}{c}0.87 \\
\pm 0.005\end{array}$ & 0.90 \\
\hline $\mathrm{S}_{3}$ & $\begin{array}{c}-0.06 \\
\pm 0.003\end{array}$ & $\begin{array}{c}0.71 \\
\pm 0.04\end{array}$ & 0.59 & $\begin{array}{c}0.08 \\
\pm 0.004\end{array}$ & $\begin{array}{c}1.04 \\
\pm 0.008\end{array}$ & 0.84 \\
\hline $\mathrm{S}_{4}$ & $\begin{array}{c}0.30 \\
\pm 0.005\end{array}$ & $\begin{array}{c}0.97 \\
\pm 0.01\end{array}$ & 0.69 & $\begin{array}{c}-0.14 \\
\pm 0.003\end{array}$ & $\begin{array}{c}0.84 \\
\pm 0.005\end{array}$ & 0.89 \\
\hline $\mathrm{S}_{5}$ & $\begin{array}{c}0.10 \\
\pm 0.006\end{array}$ & $\begin{array}{c}0.77 \\
\pm 0.02\end{array}$ & 0.44 & $\begin{array}{c}-0.01 \\
\pm 0.003\end{array}$ & $\begin{array}{c}0.94 \\
\pm 0.006\end{array}$ & 0.87 \\
\hline $\mathbf{A v g}$ & $\mathbf{0 . 1 1}$ & $\mathbf{0 . 8 0}$ & $\mathbf{0 . 6 8}$ & $\begin{array}{c}\mathbf{0 . 0 1} \\
\pm \mathbf{0 . 0 4}\end{array}$ & $\begin{array}{c}\mathbf{0 . 9 0} \\
\pm \mathbf{0 . 0 4}\end{array}$ & $\pm \mathbf{0 . 0 1}$ \\
\hline SE & $\pm \mathbf{0 . 0 6}$ & $\pm \mathbf{0 . 0 5}$ & $\pm \mathbf{0 . 0 8}$ & $\pm \mathbf{0 . 0 4}$ & \\
\hline
\end{tabular}

Table 5. Experiment 5: Linear fits of the $\Delta \mathrm{B}_{\mathrm{z}, \mathrm{c}}$ measurements and simulations across five different subjects for the two current injection profiles (R-L and A-P). The table lists the intercepts $\beta_{0}$, the slopes $\beta_{1}$, and the coefficient of determination $\mathrm{R}^{2}$ of the fitted linear regression models. For $\beta_{0}$ and $\beta_{1}$, also the standard errors are stated. The last row lists the averages across subjects, and the standard error of the averages. Most estimated slopes are lower than unity (i.e., the simulations slightly

measurements at $\mathrm{T}_{\mathrm{R}}=80 \mathrm{~ms}$ and $120 \mathrm{~ms}$ exhibit lower noise standard deviations than the measurements at $\mathrm{T}_{\mathrm{R}}=40 \mathrm{~ms}$. underestimate the $\Delta \mathrm{B}_{z, \mathrm{c}}$ ). The significance of the regression models was confirmed using F-tests, with the results being highly significant $\left(\mathrm{p}<10^{-6}\right)$ in all cases. 


\begin{tabular}{|c|c|c|c|c|c|c|}
\hline & \multicolumn{6}{|c|}{ R-L } \\
\hline & \multicolumn{3}{|c|}{ Uncorrected } & \multicolumn{3}{|c|}{ Corrected } \\
\hline & $\begin{array}{c}\boldsymbol{\beta}_{\mathbf{0}} \\
\text { in }\left[\mathrm{A} / \mathrm{m}^{2}\right]\end{array}$ & $\beta_{1}$ & $\mathbf{R}^{2}$ & $\begin{array}{c}\boldsymbol{\beta}_{\mathbf{0}} \\
\text { in }\left[\mathrm{A} / \mathrm{m}^{2}\right]\end{array}$ & $\beta_{1}$ & $\mathbf{R}^{2}$ \\
\hline $\mathrm{S}_{1}$ & $\begin{array}{c}0.021 \\
\pm 0.001\end{array}$ & $\begin{aligned} & 0.56 \\
\pm & 6.4 \cdot 10^{-3}\end{aligned}$ & 0.68 & $\begin{array}{c}0.018 \\
\pm 0.001\end{array}$ & $\begin{aligned} & 0.68 \\
\pm & 8.7 \cdot 10^{-3}\end{aligned}$ & 0.63 \\
\hline $\mathrm{S}_{2}$ & $\begin{array}{c}0.016 \\
\pm 0.001\end{array}$ & $\begin{aligned} & 0.71 \\
\pm & 6.6 \cdot 10^{-3}\end{aligned}$ & 0.75 & $\begin{array}{c}0.014 \\
\pm 0.001\end{array}$ & $\begin{aligned} & 0.78 \\
\pm & 7.5 \cdot 10^{-3}\end{aligned}$ & 0.74 \\
\hline $\mathrm{S}_{3}$ & $\begin{array}{c}0.016 \\
\pm 0.001\end{array}$ & $\begin{array}{c}0.69 \\
\pm 7.4 \cdot 10^{-3}\end{array}$ & 0.73 & $\begin{array}{c}0.007 \\
\pm 0.001\end{array}$ & $\begin{array}{c}0.89 \\
\pm 8.9 \cdot 10^{-3}\end{array}$ & 0.76 \\
\hline $\mathrm{S}_{4}$ & $\begin{array}{c}0.018 \\
\pm 0.001\end{array}$ & $\begin{array}{c}0.61 \\
\pm 10.7 \cdot 10^{-3}\end{array}$ & 0.53 & $\begin{array}{c}0.014 \\
\pm 0.001\end{array}$ & $\begin{array}{c}0.74 \\
\pm 9.2 \cdot 10^{-3}\end{array}$ & 0.69 \\
\hline $\mathrm{S}_{5}$ & $\begin{array}{c}0.019 \\
\pm 0.001\end{array}$ & $\begin{aligned} & 0.65 \\
\pm & 6.8 \cdot 10^{-3}\end{aligned}$ & 0.74 & $\begin{array}{c}0.011 \\
\pm 0.001\end{array}$ & $\begin{array}{c}0.80 \\
\pm 7.4 \cdot 10^{-3}\end{array}$ & 0.79 \\
\hline $\begin{array}{l}\text { Avg } \\
\pm \text { SE }\end{array}$ & $\begin{array}{c}0.018 \\
\pm 0.001\end{array}$ & $\begin{array}{c}0.64 \\
\pm 0.03\end{array}$ & $\begin{array}{c}0.69 \\
\pm 0.04\end{array}$ & $\begin{array}{c}0.013 \\
\pm 0.002\end{array}$ & $\begin{array}{c}\mathbf{0 . 7 8} \\
\pm \mathbf{0 . 0 3}\end{array}$ & $\begin{array}{c}0.72 \\
\pm 0.03\end{array}$ \\
\hline
\end{tabular}

735 Table 6. Experiment 5: Linear fits of the current density distributions reconstructed from 736 measurements and simulations. Listed are the results for the current injection profile R-L, for both the 737 cases with and without stray magnetic field correction. The table lists the intercepts $\beta_{0}$, the slopes $\beta_{1}$, and the coefficient of determination $\mathrm{R}^{2}$ of the fitted linear regression models. For $\beta_{0}$ and $\beta_{1}$, also the standard errors are stated. The last row lists the averages across subjects, and the standard error of the averages. The estimated slopes increase on average by 0.14 for the corrected vs. uncorrected case. Also for the corrected case, the estimated slopes are still lower than unity (i.e., the simulations underestimate the current density). The significance of the regression models was confirmed using Ftests, with the results being highly significant $\left(\mathrm{p}<10^{-6}\right)$ in all cases. 


\begin{tabular}{|c|c|c|c|c|c|c|}
\hline & \multicolumn{6}{|c|}{$A-P$} \\
\hline & \multicolumn{3}{|c|}{ Uncorrected } & \multicolumn{3}{|c|}{ Corrected } \\
\hline & $\begin{array}{c}\boldsymbol{\beta}_{\mathbf{0}} \\
\text { in }\left[\mathrm{A} / \mathrm{m}^{2}\right]\end{array}$ & $\beta_{1}$ & $\mathbf{R}^{2}$ & $\begin{array}{c}\boldsymbol{\beta}_{\mathbf{0}} \\
\text { in }\left[\mathrm{A} / \mathrm{m}^{2}\right]\end{array}$ & $\beta_{1}$ & $\mathbf{R}^{2}$ \\
\hline $\mathrm{S}_{1}$ & $\begin{array}{c}0.032 \\
\pm 0.001\end{array}$ & $\begin{array}{c}0.49 \\
\pm 7.4 \cdot 10^{-3}\end{array}$ & 0.55 & $\begin{array}{c}0.023 \\
\pm 0.001\end{array}$ & $\begin{array}{c}0.71 \\
\pm 9.2 \cdot 10^{-3}\end{array}$ & 0.62 \\
\hline $\mathrm{S}_{2}$ & $\begin{array}{c}0.022 \\
\pm 0.001\end{array}$ & $\begin{array}{c}0.67 \\
\pm 6.4 \cdot 10^{-3}\end{array}$ & 0.74 & $\begin{array}{c}0.015 \\
\pm 0.001\end{array}$ & $\begin{array}{c}0.84 \\
\pm 7.9 \cdot 10^{-3}\end{array}$ & 0.75 \\
\hline $\mathrm{S}_{3}$ & $\begin{array}{c}0.022 \\
\pm 0.001\end{array}$ & $\begin{array}{c}0.70 \\
\pm 9.0 \cdot 10^{-3}\end{array}$ & 0.65 & $\begin{array}{c}0.014 \\
\pm 0.001\end{array}$ & $\begin{array}{c}0.83 \\
\pm 10.8 \cdot 10^{-3}\end{array}$ & 0.65 \\
\hline $\mathrm{S}_{4}$ & $\begin{array}{c}0.023 \\
\pm 0.001\end{array}$ & $\begin{array}{c}0.79 \\
\pm 10.2 \cdot 10^{-3}\end{array}$ & 0.68 & $\begin{array}{c}0.009 \\
\pm 0.001\end{array}$ & $\begin{array}{c}0.84 \\
\pm 10.2 \cdot 10^{-3}\end{array}$ & 0.71 \\
\hline $\mathrm{S}_{5}$ & $\begin{array}{c}0.018 \\
\pm 0.001\end{array}$ & $\begin{aligned} & 0.67 \\
\pm & 6.5 \cdot 10^{-3}\end{aligned}$ & 0.77 & $\begin{array}{c}0.010 \\
\pm 0.001\end{array}$ & $\begin{array}{c}0.91 \\
\pm 7.6 \cdot 10^{-3}\end{array}$ & 0.82 \\
\hline $\begin{array}{l}\text { Avg } \\
\pm \text { SE }\end{array}$ & $\begin{array}{c}0.023 \\
\pm 0.002\end{array}$ & $\begin{array}{c}0.66 \\
\pm 0.05\end{array}$ & $\begin{array}{c}0.68 \\
\pm 0.04\end{array}$ & $\begin{array}{c}0.014 \\
\pm 0.003\end{array}$ & $\begin{array}{c}\mathbf{0 . 8 3} \\
\pm \mathbf{0 . 0 3}\end{array}$ & $\begin{array}{c}0.71 \\
\pm 0.04\end{array}$ \\
\hline
\end{tabular}

750 Table 7. Experiment 5: Linear fits of the current density distributions reconstructed from

751 measurements and simulations. Listed are the results for the current injection profile A-P. The

752 estimated slopes increase on average by 0.16 for the corrected vs. uncorrected case. The estimated

753 slopes are still lower than unity also for the corrected case (i.e., the simulations underestimates the

754 current density). This is similar to the results observed for current injection profile R-L. The

755 significance of the regression models was confirmed using F-tests, with the results being highly

756 significant $\left(\mathrm{p}<10^{-6}\right)$ in all cases. 
758

759

760

761

762

763

764

765

766

767

768

769

770

771

772

773

774

775

776

777

778

779

780

781

782

783

784

785

786

787

788

789

790

791

792

793

794

795

796

797

798

799

800

801

802

\section{References}

Bieri, O., Maderwald, S., Ladd, M.E., Scheffler, K., 2006. Balanced alternating steady-state elastography. Magn. Reson. Med. 55, 233-41.

Birgül, Ö., Eyüboğlu, B.M., Ider, Y.Z., 2003. Experimental results for 2D magnetic resonance electrical impedance tomography (MR-EIT) using magnetic flux density in one direction. Phys. Med. Biol. 48, 3485-3504.

Dabek, J., Kalogianni, K., Rotgans, E., van der Helm, F.C.T., Kwakkel, G., van Wegen, E.E.H., Daffertshofer, A., de Munck, J.C., 2016. Determination of head conductivity frequency response in vivo with optimized EIT-EEG. Neuroimage 127, 484-495. doi:10.1016/j.neuroimage.2015.11.023

Eyüboğlu, B.M., 2006a. Electrical Impedance Imaging, Injected Current, in: Wiley Encyclopedia of Biomedical Engineering. pp. 1195-1205.

Eyüboğlu, B.M., 2006b. Magnetic Resonance Current Density Imaging, in: Wiley Encyclopedia of Biomedical Engineering. pp. 2147-53.

Eyüboğlu, B.M., 2006c. Magnetic Resonance-Electrical Impedance Tomography, in: Wiley Encyclopedia of Biomedical Engineering. pp. 2154-62.

Faes, T.J.C., Meij, H.A. van der, Munck, J.C. de, Heethaar, R.M., 1999. The electric resistivity of human tissues (100 Hz-10 MHz): a meta-analysis of review studies. Physiol. Meas. 20, R1-R10. doi:10.1088/0967-3334/20/4/201

Göksu, C., Sadighi, M., Eroğlu, H.H., Eyüboğlu, M., 2014. Realization of Magnetic Resonance Current Density Imaging at 3 Tesla, in: Annual International Conference of the IEEE Engineering in Medicine and Biology Society - EMBC. pp. 1115-1118.

Göksu, C., Scheffler, K., Ehses, P., Hanson, L.G., Thielscher, A., 2017. Sensitivity Analysis of Magnetic Field Measurements for Magnetic Resonance Electrical Impedance Tomography (MREIT). Magn. Reson. Med. 0. doi:10.1002/mrm.26727

Haase, A., Frahm, J., Hänicke, W., Matthaei, D., 1985. 1H NMR chemical shift selective (CHESS) imaging. Phys. Med. Biol. 30, 341-344.

Han, Y.Q., Meng, Z.J., Jeong, W.C., Kim, Y.T., Minhas, A.S., Kim, H.J., Nam, H.S., Kwon, O., Woo, E.J., 2010. MREIT conductivity imaging of canine head using multi-echo pulse sequence. J. Phys. Conf. Ser. 224, 12078. doi:10.1088/1742-6596/224/1/012078

Huang, Y., Liu, A.A., Lafon, B., Friedman, D., Dayan, M., Wang, X., Bikson, M., Doyle, W.K., Devinsky, O., Parra, L.C., 2017. Measurements and models of electric fields in the in vivo human brain during transcranial electric stimulation. Elife 6, e18834. doi:10.7554/eLife.18834

Ida, M., Wakayama, T., Nielsen, M.L., Abe, T., Grodzki, D.M., 2015. Quiet T1-Weighted Imaging Using PETRA : Initial Clinical Evaluation in Intracranial Tumor Patients. J. Magn. Reson. Imaging 41, 447-453. doi:10.1002/jmri.24575

Ider, Y.Z., Birgül, Ö., 1998. Use of the magnetic field generated by the internal distribution of injected currents for Electrical Impedance Tomography (MR-EIT). ELEKTRIK 6, 215-225.

Ider, Y.Z., Birgül, Ö., Oran, Ö.F., Arıkan, O., Hamamura, M.J., Müftüler, T., 2010. Fourier transform magnetic resonance current density imaging ( FT-MRCDI ) from one component of magnetic flux density. Phys. Med. Biol. 55, 3177-3199. doi:10.1088/0031-9155/55/11/013

Jaynes, E.T., 1955. Matrix treatment of nuclear induction. Phys. Rev. 98, 1099-1105.

Jeon, K., Minhas, A.S., Kim, Y.T., Jeong, W.C., Kim, H.J., Kang, B.T., Park, H.M., Lee, C.-O., Seo, J.K., Woo, E.J., 2009. MREIT conductivity imaging of the postmortem canine abdomen using CoReHA. Physiol. Meas. 30, 957-66. doi:10.1088/0967-3334/30/9/007 
Jeong, W.C., Kim, Y.T., Minhas, a S., Lee, T.H., Kim, H.J., Nam, H.S., Kwon, O., Woo, E.J., 2010. In vivo conductivity imaging of human knee using $3 \mathrm{~mA}$ injection current in MREIT. J. Phys. Conf. Ser. 224, 12148. doi:10.1088/1742-6596/224/1/012148

Jog, M. V, Smith, R.X., Jann, K., Dunn, W., Lafon, B., Truong, D., Wu, A., Parra, L., Bikson, M., Wang, D.J.J., 2016. In-vivo Imaging of Magnetic Fields Induced by Transcranial Direct Current Stimulation (tDCS) in Human Brain using MRI. Sci. Rep. 6, 34385. doi:10.1038/srep34385

Joy, M.L.G., 2004. MR current density and conductivity imaging: the state of the art., in: Conf Proc IEEE Eng Med Biol Soc. pp. 5315-9. doi:10.1109/IEMBS.2004.1404484

Kasinadhuni, A.K., Indahlastari, A., Chauhan, M., Scär, M., Mareci, T.H., Sadleir, R.J., 2017. Imaging of current flow in the human head during transcranial electrical therapy. Brain Stimul. 10, 764-772. doi:10.1016/j.brs.2017.04.125

Kim, H.J., Kim, Y.T., Minhas, A.S., Jeong, W.C., Woo, E.J., 2009. In Vivo High-Resolution Conductivity Imaging of the Human Leg Using MREIT : The First Human Experiment 28, $1681-1687$.

Kim, H.J., Oh, T.I., Kim, Y.T., Lee, B. Il, Woo, E.J., Seo, J.K., Lee, S.Y., Kwon, O., Park, C., Kang, B.T., Park, H.M., 2008. In vivo electrical conductivity imaging of a canine brain using a $3 \mathrm{~T}$ MREIT system. Physiol. Meas. 29, 1145-55. doi:10.1088/0967-3334/29/10/001

Kim, Y.T., Minhas, A.S., Meng, Z., Kim, H.J., Woo, E.J., 2011. Conductivity imaging of human lower extremity using MREIT with multi-echo pulse sequence and $3 \mathrm{~mA}$ imaging current. 2011 8th Int. Symp. Noninvasive Funct. Source Imaging Brain Hear. 2011 8th Int. Conf. Bioelectromagn. 48-52. doi:10.1109/NFSI.2011.5936818

Kwon, O.I., Sajib, S.Z., Sersa, I., Oh, T.I., Jeong, W.C., Kim, H.J., Woo, E.J., 2016. Current Density Imaging During Transcranial Direct Current Stimulation Using DT-MRI and MREIT: Algorithm Development and Numerical Simulations. IEEE Trans Biomed Eng 63, 168-175. doi:10.1109/TBME.2015.2448555

Lee, C., Jeon, K., Ahn, S., Kim, H.J., Woo, E.J., Member, S., 2011. Ramp-Preserving Denoising for Conductivity Image Reconstruction in Magnetic Resonance Electrical Impedance Tomography 58, 2038-2050.

Lee, H., Jeong, W.C., Kim, H.J., Woo, E.J., Park, J., 2016. Alternating steady state free precession for estimation of current-induced magnetic flux density: A feasibility study. Magn. Reson. Med. 75, 2009-2019.

Meng, Z., Sajib, S.Z., Chauhan, M., Jeong, W.C., Kim, Y.T., Kim, H.J., Woo, E.J., 2012. Improved conductivity image of human lower extremity using MREIT with chemical shift artifact correction. Biomed. Eng. Lett. 2, 62-68. doi:10.1007/s13534-012-0052-0

Minhas, A.S., Woo, E.J., Sadleir, R., 2010. Simulation of MREIT using balanced steady state free precession (b-SSFP) pulse sequence. J. Phys. Conf. Ser. 224, 12019. doi:10.1088/17426596/224/1/012019

Miranda, P.C., 2013. Physics of effects of transcranial brain stimulation. Handb. Clin. Neurol. 116, 353-366.

Mosher, J.C., Leahy, R.M., Lewis, P.S., 1999. EEG and MEG: Forward Solutions for Inverse Methods. IEEE Trans. Biomed. Eng. 46, 245-259.

Nam, H.S., Kwon, O.I., 2010. Optimization of multiply acquired magnetic flux density B(z) using ICNE-Multiecho train in MREIT. Phys. Med. Biol. 55, 2743-59.

Nicholson, P.W., 1965. Specific impedance of cerebral white matter. Exp. Neurol. 13, 386-401. doi:10.1016/0014-4886(65)90126-3

Nitsche, M.A., Paulus, W., 2000. Excitability changes induced in the human motor cortex by weak 
transcranial direct current stimulation. J. Physiol. 527, 633-639.

Oh, S.H., Lee, B. Il, Woo, E.J., Lee, S.Y., Cho, M.H., Kwon, O., Seo, J.K., 2003. Conductivity and current density image reconstruction using harmonic Bz algorithm in magnetic resonance electrical impedance tomography. Phys. Med. Biol. 48, 3101-3116. doi:10.1088/00319155/48/19/001

Oh, S.H., Lee, B. Il, Woo, E.J., Lee, S.Y., Kim, T.-S., Kwon, O., Seo, J.K., 2005. Electrical conductivity images of biological tissue phantoms in MREIT. Physiol. Meas. 26, S279-88. doi:10.1088/0967-3334/26/2/026

Oostenveld, R., Fries, P., Maris, E., Schoffelen, J.M., 2011. FieldTrip: Open source software for advanced analysis of MEG, EEG, and invasive electrophysiological data. Comput. Intell. Neurosci. 2011. doi:10.1155/2011/156869

Opitz, A., Falchier, A., Yan, C.-G., Yeagle, E.M., Linn, G.S., Megevand, P., Thielscher, A., Deborah A., R., Milham, M.P., Mehta, A.D., Schroeder, C.E., 2016. Spatiotemporal structure of intracranial electric fields induced by transcranial electric stimulation in humans and nonhuman primates. Sci. Rep. 6, 31236. doi:10.1038/srep31236

Opitz, A., Windhoff, M., Heidemann, R.M., Turner, R., Thielscher, A., 2011. How the brain tissue shapes the electric field induced by transcranial magnetic stimulation. Neuroimage 58, 849-859. doi:10.1016/j.neuroimage.2011.06.069

Park, C., Lee, B. Il, Kwon, O.I., 2007. Analysis of recoverable current from one component of magnetic flux density in MREIT and MRCDI. Phys. Med. Biol. 52, 3001-13. doi:10.1088/0031$9155 / 52 / 11 / 005$

Sadighi, M., Göksu, C., Eyüboğlu, B.M., 2014. J-based Magnetic Resonance Conductivity Tensor Imaging (MRCTI) at 3 T. Annu. Int. Conf. IEEE Eng. Med. Biol. Soc. - EMBC 1139-1142.

Sadleir, R.J., Grant, S.C., Silver, X., Zhang, S.U., Woo, E.J., Lee, S.Y., Kim, T.S., Oh, S.H., Lee, B.I., Seo, J.K., 2005. Magnetic Resonance Electrical Impedance Tomography ( MREIT ) at 11 Tesla Field Strength : Preliminary Experimental Study 7, 340-343.

Sajib, S.Z.K., Kim, H.J., Kwon, O.I., Woo, E.J., 2012. Regional absolute conductivity reconstruction using projected current density in MREIT. Phys. Med. Biol. 57, 5841-5859. doi:10.1088/0031$9155 / 57 / 18 / 5841$

Saturnino, G.B., Antunes, A., Thielscher, A., 2015. On the importance of electrode parameters for shaping electric field patterns generated by tDCS. Neuroimage 120, 25-35. doi:10.1016/j.neuroimage.2015.06.067

Scheffler, K., Maderwald, S., Ladd, M.E., Bieri, O., 2006. Oscillating steady states. Magn. Reson. Med. 55, 598-603.

Scott, G.C., Joy, M.L.G., Armstrong, R.L., Henkelman, R.M., 1992. Sensitivity of magneticresonance current-density imaging. J. Magn. Reson. 97, 235-254.

Scott, G.C., Joy, M.L.G., Armstrong, R.L., Henkelman, R.M., 1991. Measurement of nonuniform current density by magnetic resonance. IEEE Trans. Med. Imaging 10, 362-374.

Seo, J.K., Woo, E.J., 2011. Magnetic Resonance Electrical Impedance Tomography. Soc. Ind. Appl. Math. 53, 40-68.

Seo, J.K., Yoon, J., Woo, E.J., Kwon, O., 2003. Reconstruction of Conductivity and Current Density Images Using Only One Component of Magnetic Field Measurements. IEEE Trans. Biomed. Eng. 50, 1121-1124.

Tadel, F., Baillet, S., Mosher, J.C., Pantazis, D., Leahy, R.M., 2011. Brainstorm: A User-Friendly Application for MEG/EEG Analysis. Comput. Intell. Neurosci. 2011, 1-13. doi:10.1155/2011/879716 
Thielscher, A., Antunes, A., Saturnino, G.B., 2015. Field modeling for transcranial magnetic stimulation: A useful tool to understand the physiological effects of TMS?, in: Proceedings of the Annual International Conference of the IEEE Engineering in Medicine and Biology Society, EMBS. pp. 222-225. doi:10.1109/EMBC.2015.7318340

Thielscher, A., Opitz, A., Windhoff, M., 2011. Impact of the gyral geometry on the electric field induced by transcranial magnetic stimulation. Neuroimage 54, 234-243.

Utz, K.S., Dimova, V., Oppenländer, K., Kerkhoff, G., 2010. Electrified minds: Transcranial direct current stimulation (tDCS) and Galvanic Vestibular Stimulation (GVS) as methods of noninvasive brain stimulation in neuropsychology-A review of current data and future implications. Neuropsychologia 48, 2789-2810.

Walsh, D.O., Gmitro, A.F., Marcellin, M.W., 2000. Adaptive reconstruction of phased array MR imagery. Magn. Reson. Med. 43, 682-690.

Woo, E.J., Lee, S.Y., Mun, C.W., 1994. Impedance Tomography Using Internal Current Density Distribution Measured by Nuclear Magnetic Resonance. SPIE 2299, 377-385.

Woo, E.J., Seo, J.K., 2008. Magnetic resonance electrical impedance tomography (MREIT) for highresolution conductivity imaging. Physiol. Meas. 29, R1-26. doi:10.1088/0967-3334/29/10/R01 
MESE

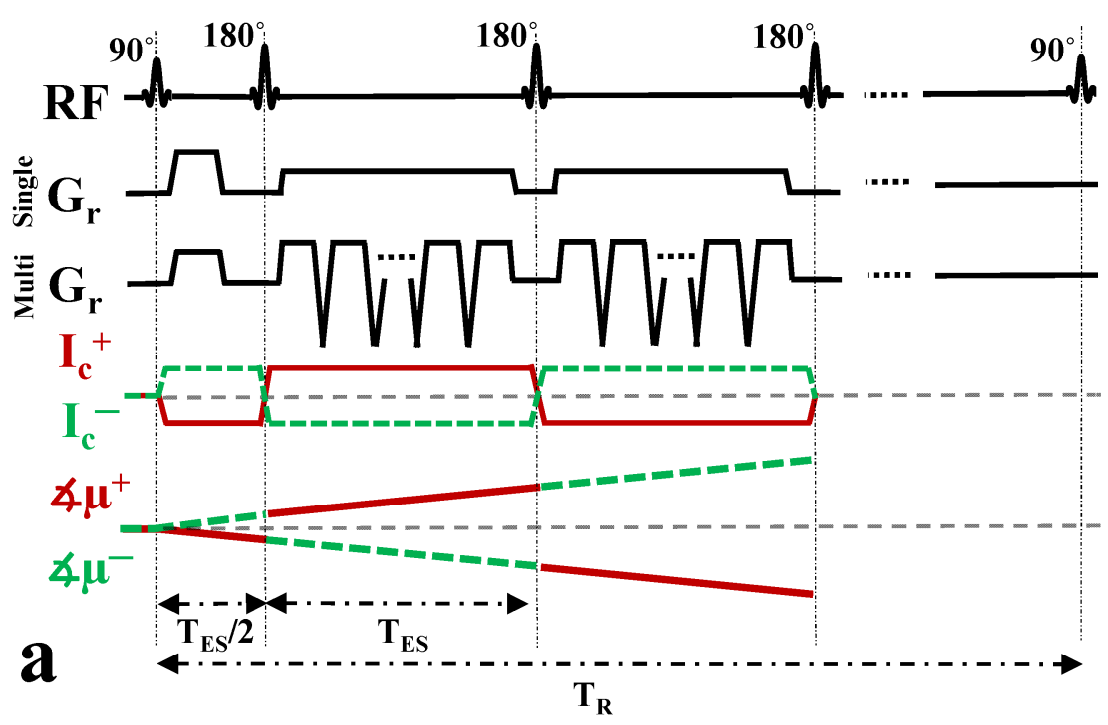

SSFP-FID

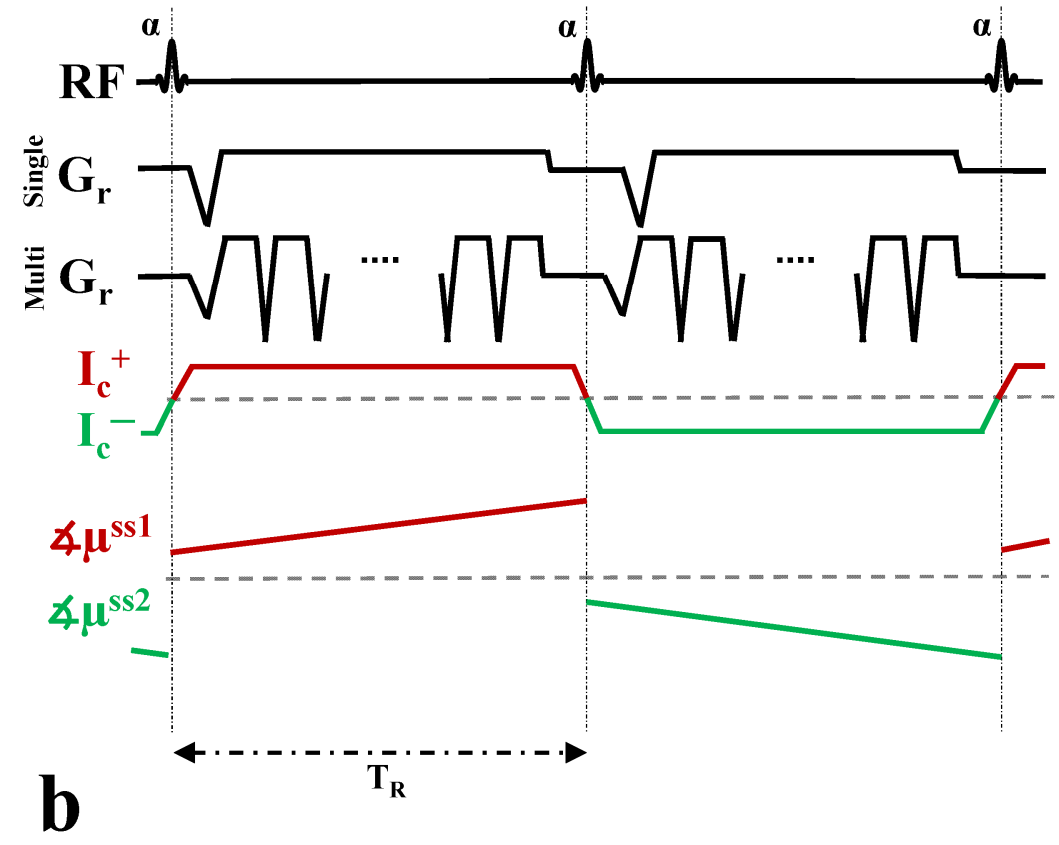




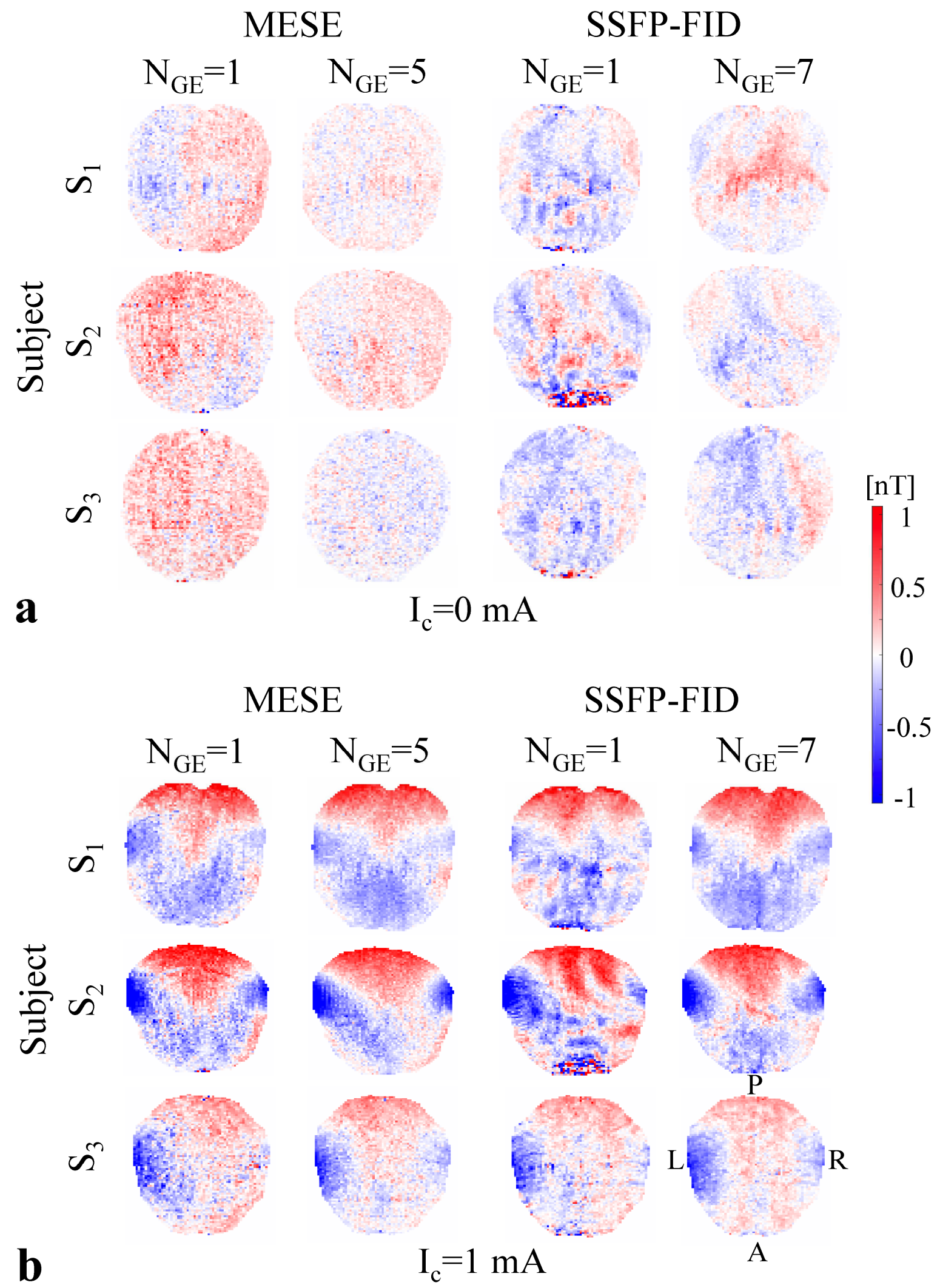




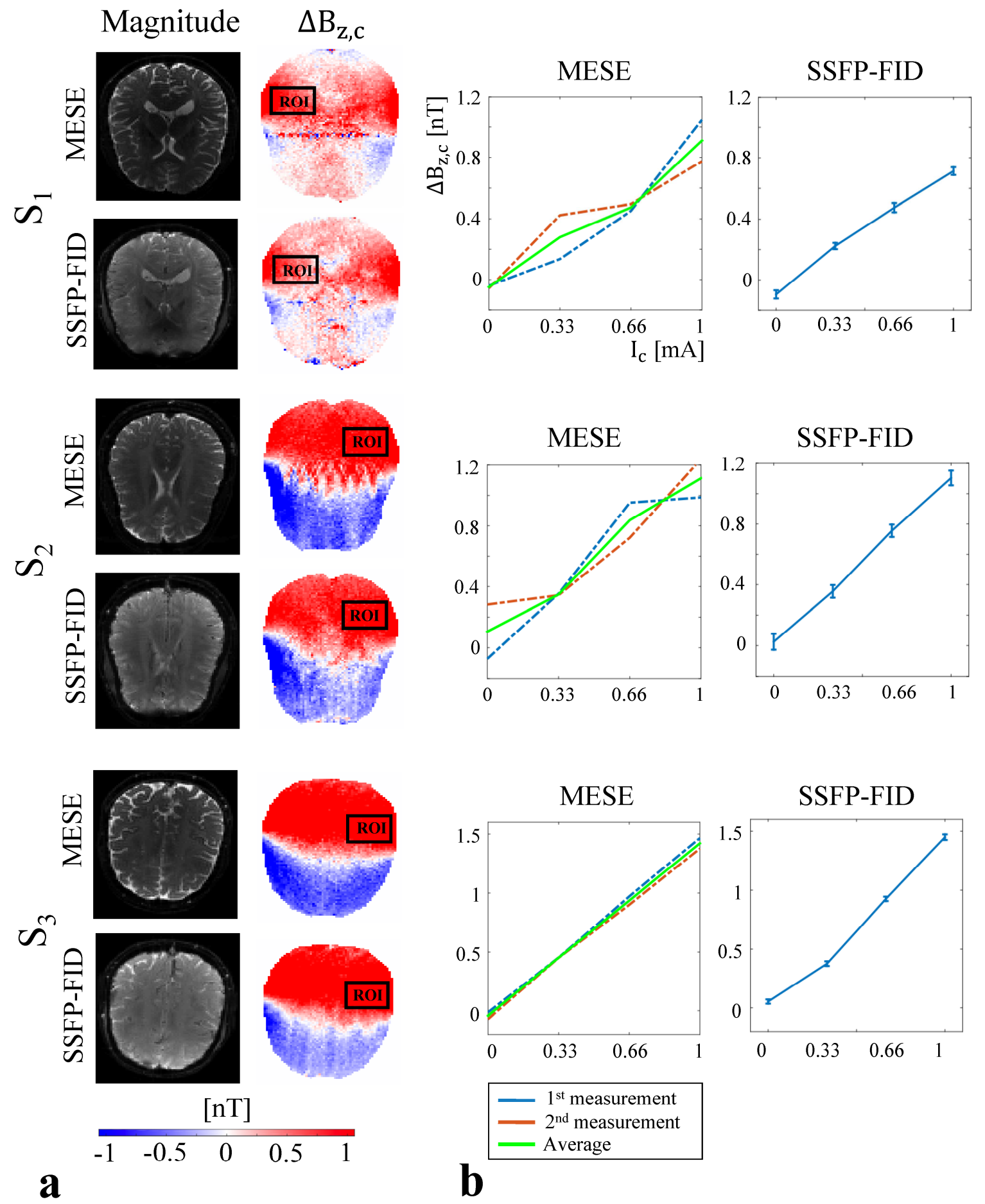




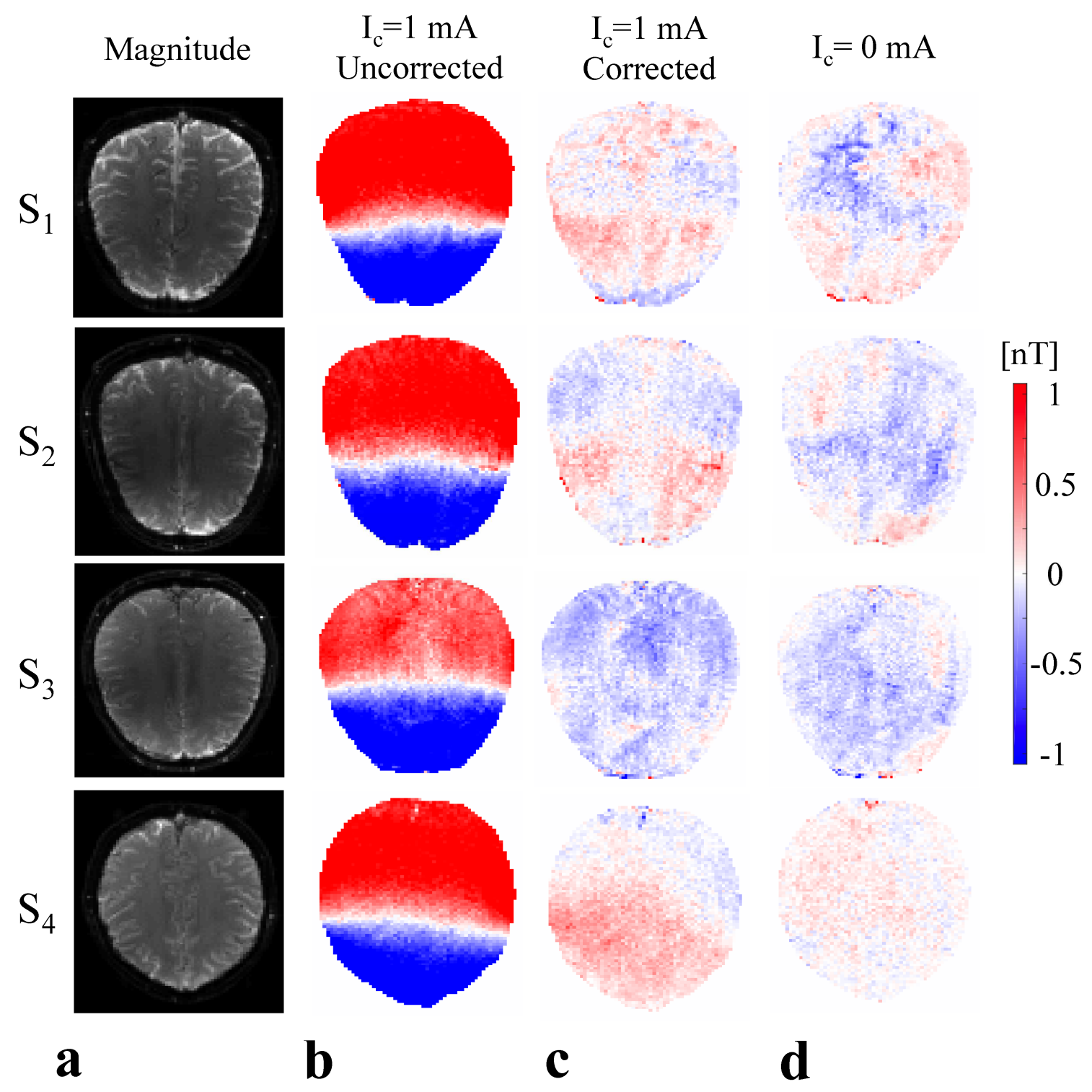




$$
\mathrm{I}_{\mathrm{c}}=0 \mathrm{~mA}
$$
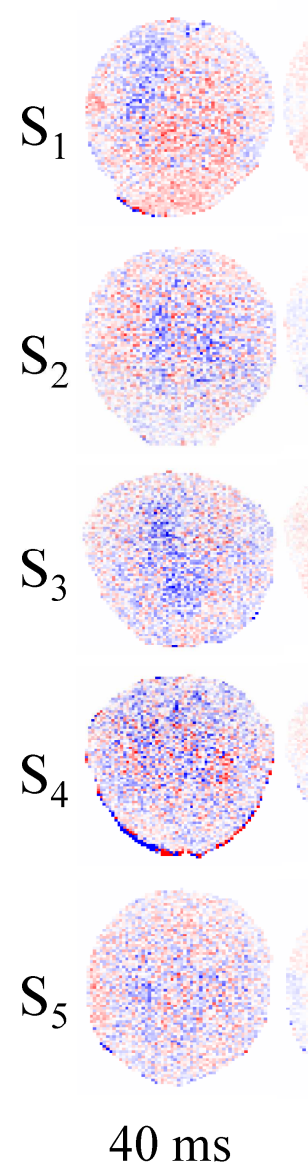

a
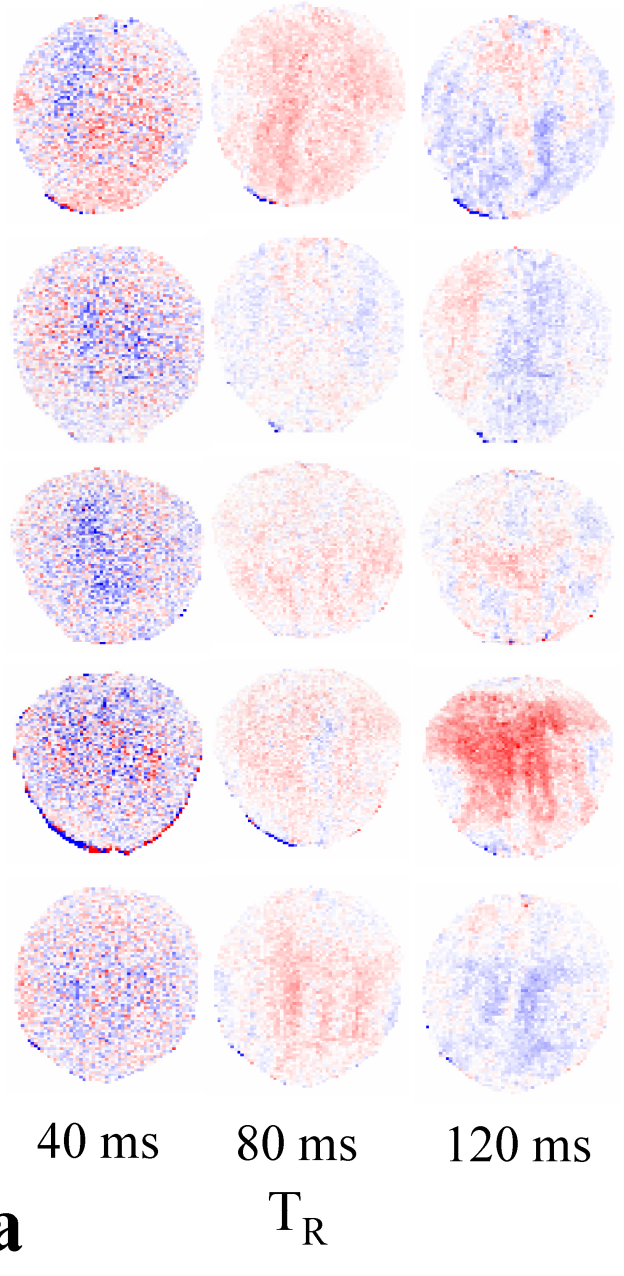

$$
\mathrm{I}_{\mathrm{c}}=1 \mathrm{~mA}
$$
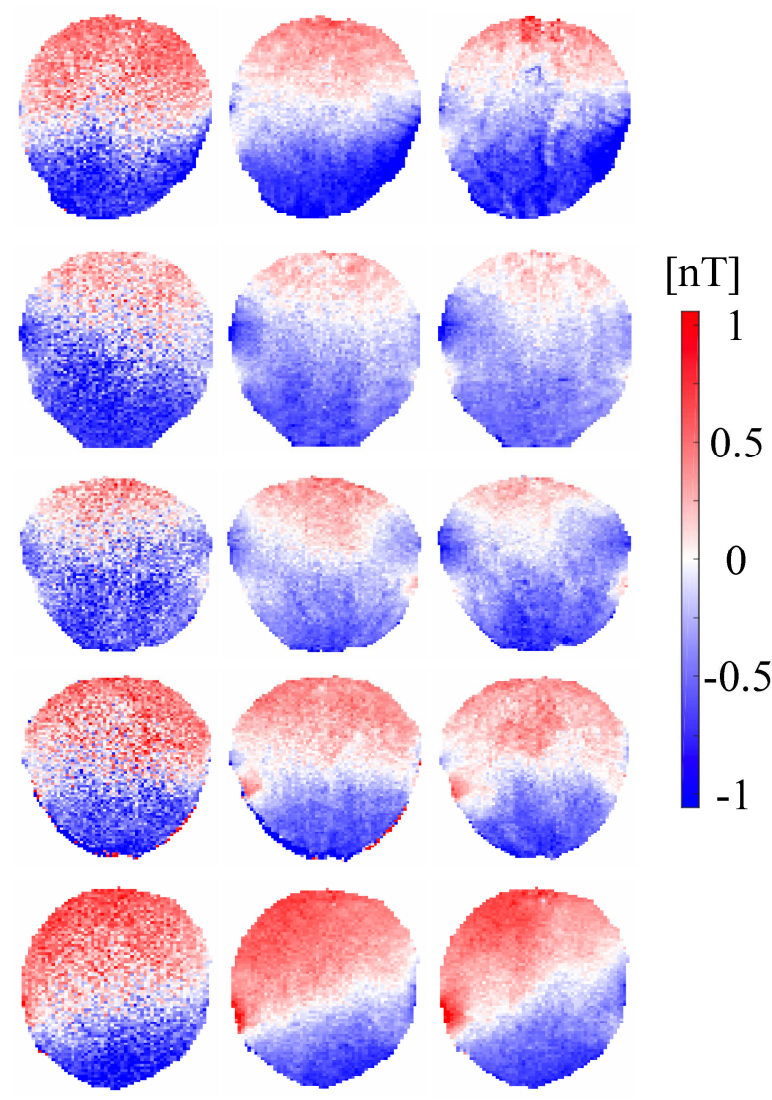

$40 \mathrm{~ms} \quad 80 \mathrm{~ms} \quad 120 \mathrm{~ms}$

b $\quad \mathrm{T}_{\mathrm{R}}$

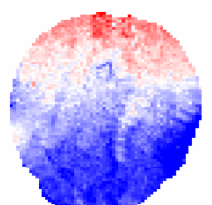

0.5

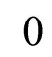

$-0.5$ 
Magnitude
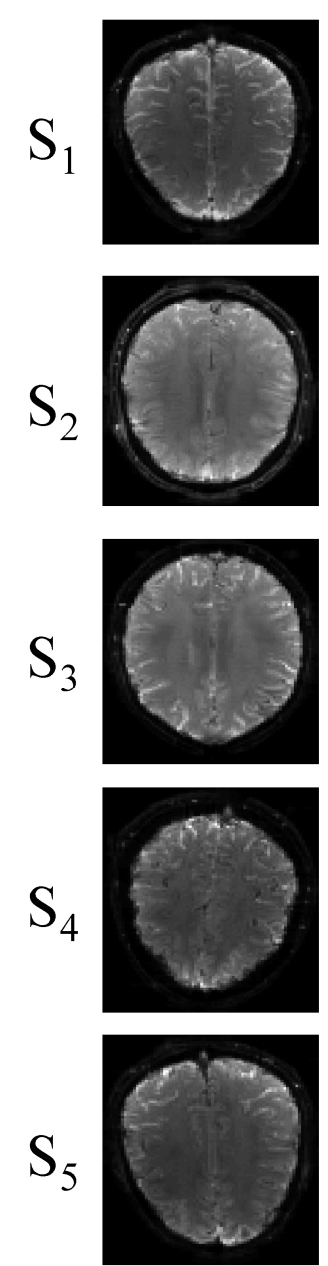

a
$\mathrm{I}_{\mathrm{c}}=1 \mathrm{~mA}$

Uncorrected
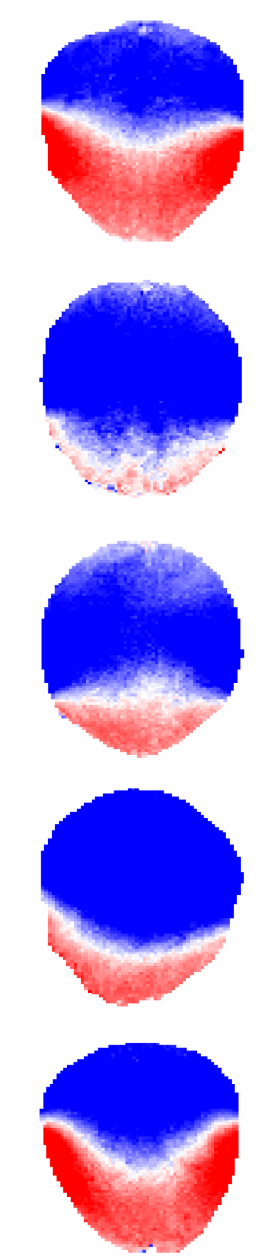

b $^{\mathrm{R}-\mathrm{L}}$
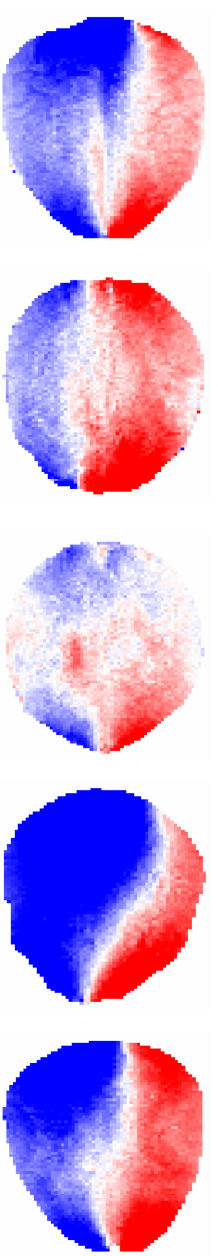

A-P

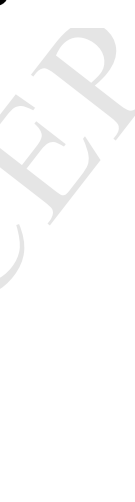




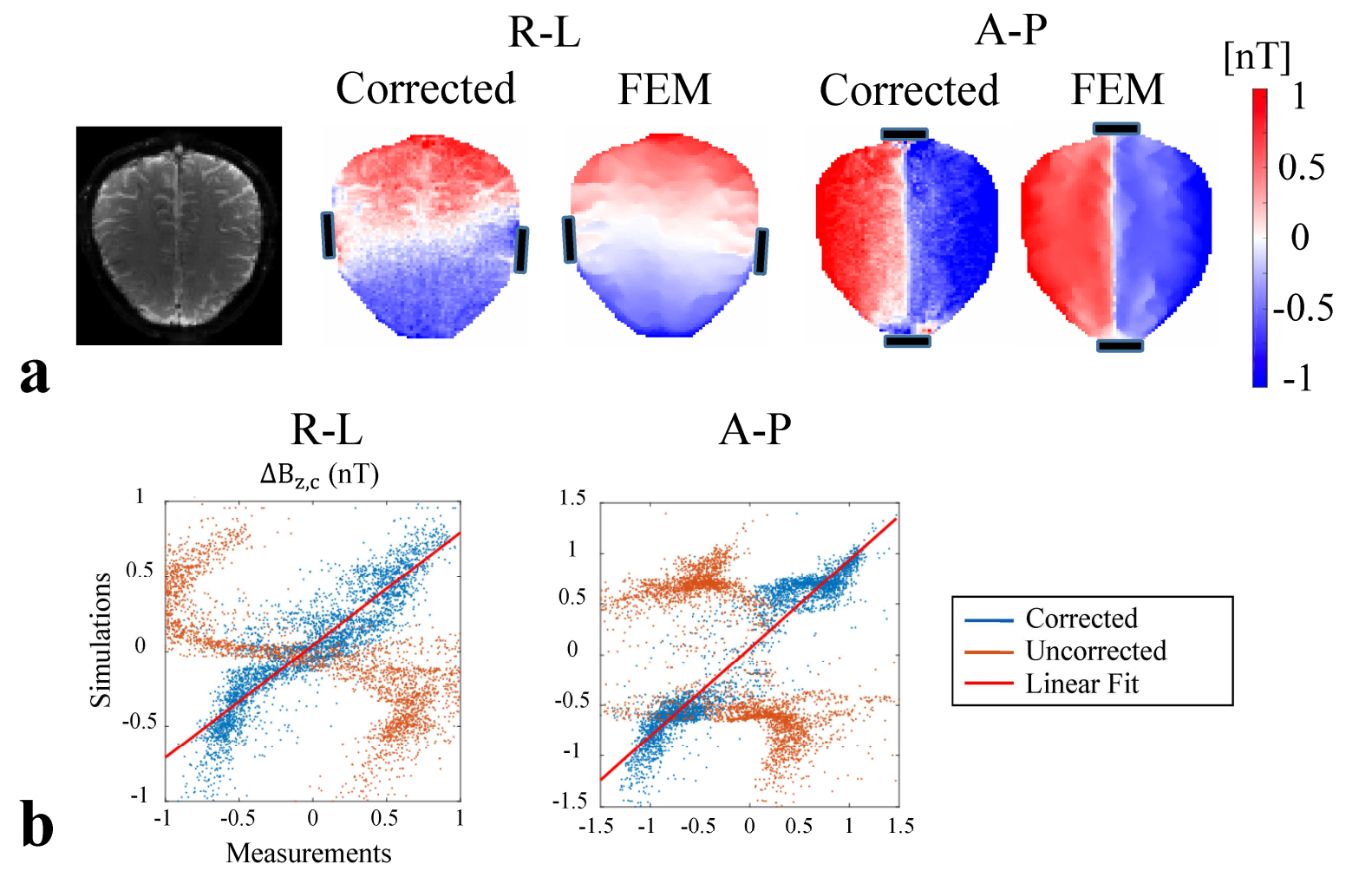




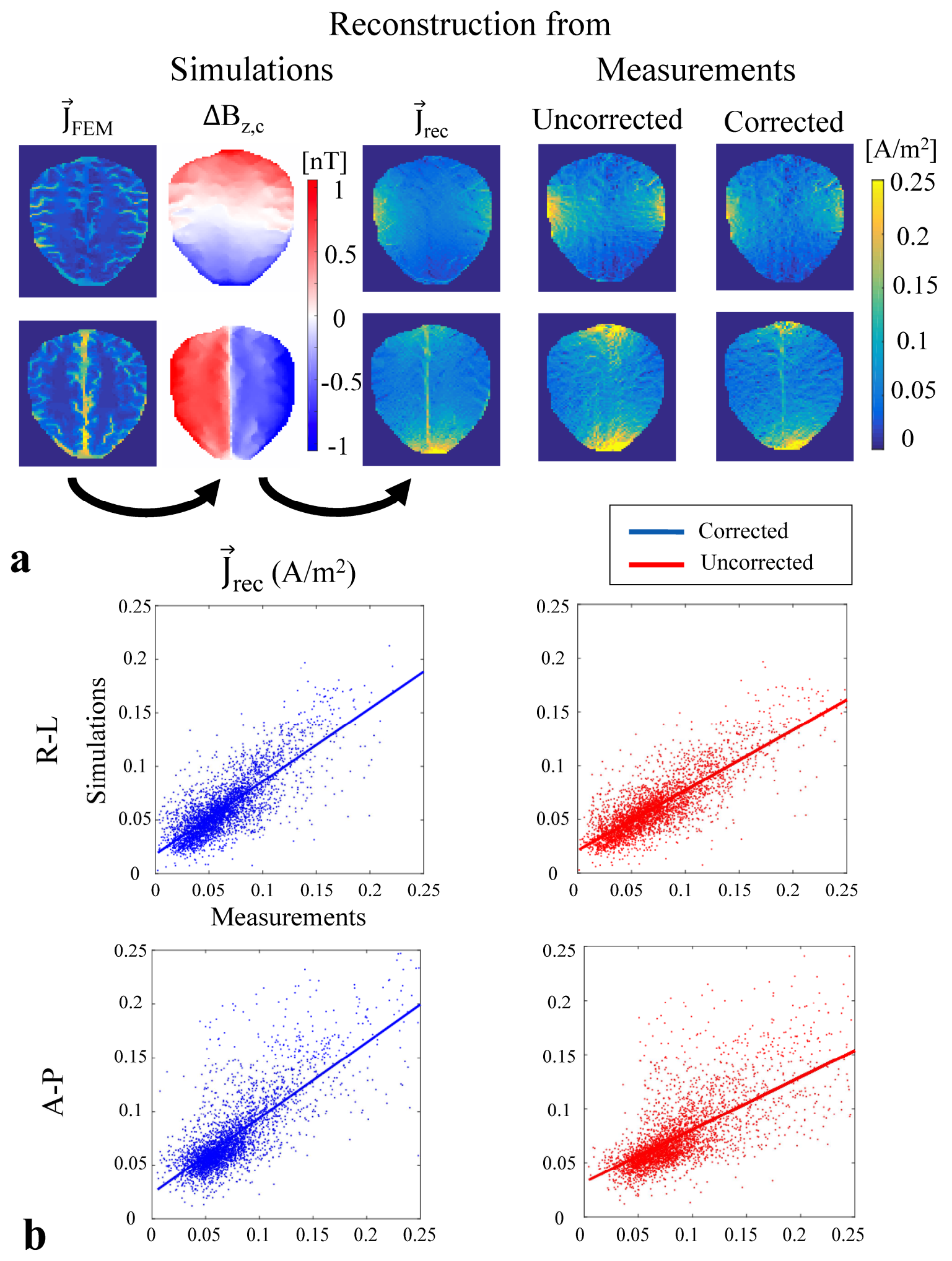

